\title{
Thermal properties of the valence electrons in alkali metal clusters ${ }^{\star}$
}

\author{
M. Brack ${ }^{1}$, O. Genzken ${ }^{1}$, and K. Hansen ${ }^{2}$ \\ ${ }^{1}$ Institute for Theoretical Physics, University of Regensburg, W-8400 Regensburg, Federal Republic of Germany \\ ${ }^{2}$ The Niels Bohr Institute, Blegdamsvej 17, DK-2100 Copenhagen, Denmark
}

Received 16 January 1991; final version 2 April 1991

\begin{abstract}
The finite-temperature density functional approach is applied for the first time to calculate thermal properties of the valence electron system in metal clusters using the spherical jellium model. Both the canonical and the grand canonical formalism are applied and their differences are discussed. We study the temperature dependence of the total free energy $F(N)$ (including a contribution from the ionic jellium background) for spherical neutral clusters containing $N$ atoms. We investigate, in particular, its first and second differences, $\Delta_{1} F=F(N-1)-F(N)$ and $\Delta_{2} F=F(N+1)$ $+F(N-1)-2 F(N)$, and discuss their possible relevance for the understanding of the mass abundance spectra observed in cluster production experiments. We show that the typical enhancement of magic spherical-shell clusters with $N=8,20,34,40,58,92,138,186,254,338,398$, $440,508,612 \ldots$, most of which are well established experimentally, is decreasing rather fast with increasing temperature $T$ and cluster size $N$. We also present electronic entropies and specific heats of spherical neutral clusters. The Koopmans theorem and related approximations for calculating $\Delta_{1} F$ and $\Delta_{2} F$ at $T>0$ are discussed.
\end{abstract}

PACS: $36.40 .+\mathrm{d} ; 31.20$. Sy; 05.30. $-\mathrm{d} ; 65.50 .+\mathrm{m}$

\section{Introduction}

Metal clusters offer on opportunity for studying interesting size and shell effects that are typical of finite Fermion systems. In particular, an enhanced stability of clusters with the 'magic' numbers of atoms ${ }^{1}$ $N=8,20,40,58,92$ has been observed by many groups

\footnotetext{
* Work partially supported by the Danish Natural Science Research Council and by Deutsche Forschungsgemeinschaft

1 We shall limit ourselves here to neutral elusters of monovalent atoms
}

(see [1] for recent reviews of expermential results). This stability can be explained in terms of a shell model for the valence electrons which move in an external field created by the ions. One of the most striking indications of the validity of the shell model is the coincidence of the steps in the first differences $\Delta_{1} E(N)$, or the peaks in the second differences $\Delta_{2} E(N)$, of the total energy $E(N)$ of neutral clusters

$$
\begin{aligned}
& \Delta_{1} E(N)=E(N-1)-E(N), \\
& \Delta_{2} E(N)=E(N+1)+E(N-1)-2 E(N),
\end{aligned}
$$

with similar features observed experimentally in mass abundance spectra and their logarithmic derivatives, at magic numbers corresponding to spherical closed shells. Steps are also observed in the electronic ionization potentials at the same magic numbers, confirming the assumption that the shell structure in the total energy is dominated by the valence electrons.

Microscopic calculations of self-consistent potentials for the electrons, taking into account their mutual Coulomb interaction, have been performed in the so-called jellium background model [2] within the Kohn-Sham density functional formalism [3]. However, these calculations - and many others since - have been performed for the ground state at zero temperature, $T=0$. On the other hand, in most experiments the clusters are produced at temperatures up to several hundred Kelvin [1], and one has to raise the question to what extent the temperature averaging of the single-particle structure might affect these results. At first sight, one would expect that a temperature of a few hundred Kelvin (i.e., a few tens of millielectronvolt) should be negligible in view of the typical major shell spacings of $\simeq 0.5-1 \mathrm{eV}$ of the electron levels in the smaller clusters. However, as we shall see, the first and second differences of the total free energy $F(N)$ with respect to the atomic number $N$ are very sensitive to temperature due to the high degeneracy of the electronic single-particle level spectrum around the magic shell closures. Bulk properties, such as total binding en- 
ergies and frequencies of collective dipole excitations of the electrons, are much less affected by temperature.

In this paper we shall present detailed selfconsistent microscopic calculations of the electronic structure of spherical metal clusters at finite temperatures. Some first results of our calculations were presented at a recent conference [4]. Our formalism is based on the $T>0$ extension of the Kohn-Sham method [5] which was originally formulated for grand canonical ensembles where the particle number is conserved only on the average. For calculations of thermal properties of a macroscopic object, the choice of ensemble is merely a matter of convenience. This is, however, no longer true in small systems where the properties under study, in particular properties affected strongly by shell structure, can change significantly within the range of a typical particle number fluctuation. For such systems a canonical description is a priori more appropriate, and the grand canonical description cannot be expected to lead to the same results.

The subsystem of valence electrons in metallic clusters represent, in fact, an ideal example of a canonical ensemble. Due to the large difference between the energy of the vibrational quanta of the positive ions and the Fermi energy, only a very minute, but finite, amount of the total thermal energy of a typical cluster will be carried by the electrons. The valence electrons will effectively be embedded in a heat bath, even for a system of free clusters. Consequently, the canonical partition function is expected to give a much better description of the thermal properties of the electronic system than either the microcanonical or the grand canonical partition functions. Since the density functional formalism also applies to canonical ensembles (see Evans [5]), we shall study here both the canonical and the grand canonical approach and compare their results systematically and carefully.

The explicit treatment of the thermal properties of the positive ionic cores is largely irrelevant for the discussion here, because all properties are assumed to change smoothly with the number of ions present in the cluster, such that the observed shell-like deviations from a smooth behaviour must be attributed to the valence electrons. This is consistent with the very idea behind the jellium model approximation which we are using: the geometrical structure of the positive ions is ignored and replaced by a uniform charge background ('jellium'). A finite temperature should only render this assumption more correct: the ensemble averaging over a slow thermal motion of the ions is likely to be equivalent to an averaging over their geometrical configurations. Thus, in the jellium model, the total (free) energy of a cluster (and quantities derived from it) only contains contributions of the ions in an averaged form. In this crude model, it would make little sense to introduce an explicit temperature dependence of the jellium density. The essential point is to include the thermal motion of the ions in terms of a heat bath with $T>0$.

Besides providing a heat bath for the electrons, the finite temperature of the ionic cores has one more consequence: namely to render the cluster unstable. Any cluster with a total excitation energy exceeding the energy needed to evaporate one atom (or, in principle, a particle of any kind) will - given sufficient time - decay. Although the mass abundance spectra display peaks near the shell closings, it is therefore not clear that measuring these spectra corresponds to a sampling of an equilibrium ensemble. Since production and sampling of the spectra experimentally is separated by at least some microseconds, sufficient time is available for substantial changes of the original abundances through evaporation. This process will also tend to increase the number of closedshell clusters due to the strong dependence of the evaporation rate on the dissociation energy. Therefore, peaks in the observed abundance spectra can be associated with shell closures even without invoking thermal equilibrium. The pronounced asymmetry of the mass spectra around magic shell closings (see, e.g., [1]) may, in fact, be taken as an indication of a significant amount of evaporation after production and before mass selection.

A semi - quantitiative comparison of observed abundance spectra with preliminary results of our calculations has been quite encouraging [6]. A more detailed comparison would necessitate the inclusion of deformation effects for clusters in the regions between the filled spherical shells. Nevertheless, we believe it to be instructive to study the properties of the electronic subsystem of individual spherical metal clusters in a thermal equilibrium situation as functions of size and temperature. Models for local chemical equilibrium or for evaporation from an initially hot ensembly may be subsequently developed. There, the free energy $F(N)$ and its first and second differences will be needed as an important input.

In Sect. 2 we present the density functional formalism for $T>0$ in the spherical jellium model. In Sect. 3 we compare the grand canonical and the canonical results for some crucial quantities and further discuss useful approximations. Section 4 is devoted to a discussion of the Koopmans theorem and related approximations which might be used to calculate $\Delta_{1} F$ and $\Delta_{2} F$ in a purely noninteracting particle picture. In Sect. 5 , we present some typical results over a large range of cluster sizes and temperatures.

\section{Kohn-Sham formalism for the jellium model at finite temperature}

\subsection{Energy functional and variational equations}

We employ the spherical jellium model [2] in which the charges of the ions (i.e. atoms minus valence electrons) are uniformly spread out over the volume of a sphere of radius $R_{I}=r_{s} N^{1 / 3}$, where $N$ is the number of ions, $r_{s}=\left(\frac{4 \pi}{3} \rho_{l}\right)^{-1 / 3}$ is the Wigner-Seitz radius characterizing the metal, and $\rho_{l}$ its density. As we have discussed in the introduction, it would not make much sense to introduce any explicit temperature dependence of the jellium density. We therefore keep the value of $r_{s}$ fixed for all temperatures.

The jellium sphere creates an external attractive potential $V_{I}(r)$ for the electrons. According to Mermin [5] and Evans [5], the Helmholtz free energy $F$ of the cluster 
is a functional of the local density $\rho(r)$ of the electrons:

$F=F[\rho]=U[\rho]-T S[\rho]$.

$U$ is the total internal energy, $S$ the entropy and $T$ the temperature ${ }^{2}$. Following the Kohn-Sham procedure [3], we introduce a non-interacting free kinetic energy $G_{s}[\rho]$

$G_{s}[\rho]=E_{s}^{\mathrm{kin}}[\rho]-T S_{s}[\rho]$,

where $E_{s}^{\mathrm{kin}}$ and $S_{s}$ are, in the standard notation, the kinetic energy and entropy, respectively, of a non-interacting system of electrons having the density $\rho(r)$. The total free energy of a cluster is then

$$
\begin{aligned}
F[\rho]=G_{s}[\rho] & +\int\left\{V_{I}(r) \rho(r)\right. \\
& +\frac{1}{2} \rho(r)\left[e^{2} \int \frac{\rho\left(r^{\prime}\right)}{\left|\mathbf{r}-\mathbf{r}^{\prime}\right|} \mathrm{d}^{3} r^{\prime}\right] \\
& \left.+\mathscr{F}_{x c}[\rho]\right\} \mathrm{d}^{3} r+E_{I} .
\end{aligned}
$$

Hereby $V_{I}(r)$ and $E_{I}$ are the potential and the electrostatic energy, respectively, of the ionic jellium background; the second term under the integral is the Hartree Coulomb energy of the electrons, and $\mathscr{F}_{x c}[\rho]$ is the exchange and correlation free energy density functional. We stress again here that $F$ contains the energy of the ions only in a very crude schematic way through the jellium background density; this contribution $E_{I}$ varies smoothly with $N$ and any shell structure effects in $F(N)$ will be due to the electrons. Similarly, the entropy associated with the ionic motion is assumed to be a smooth function of $N$; its contribution to $F(N)$ is disregarded in the following.

Next, we write $[3,5]$ the local density $\rho(r)$ of the electrons in terms of auxiliary single-particle wavefunctions $\varphi_{i}$ and finite-temperature occupation numbers $n_{i}$ as

$\rho(r)=\sum_{i}\left|\varphi_{i}(\mathbf{r})\right|^{2} n_{i}, \quad \int \rho(r) \mathrm{d}^{3} r=\sum_{i} n_{i}=N$.

and the non-interacting kinetic energy as

$E_{s}^{\mathrm{kin}}[\rho]=\frac{\hbar^{2}}{2 m} \int \sum_{i}\left|\nabla \varphi_{i}(\mathbf{r})\right|^{2} n_{i} \mathrm{~d}^{3} r$

In all sums over $i$, we shall count the degenerate singleparticle states separately, so that $0 \leqq n_{i} \leqq 1$. In principle, these sums include also an integration over the positive energy states in the particle continuum. In practice, however, we shall limit our temperatures such that the $n_{i}$ become negligible in the continuum, in order that a static equilibrium approach be justified at all.

Varying the free energy (4) with respect to the singleparticle wavefunctions $\varphi_{i}^{*}(\mathbf{r})$ leads to the usual KohnSham (KS) equations

\footnotetext{
We put the Boltzman constant $k \equiv 1$ and measure the temperature alternatively in degrees Kelvin (K) or in energy units: $1 \mathrm{Ry}=13.606 \mathrm{eV}=15.789 \cdot 10^{4} \mathrm{~K}$
}

$\left\{\hat{T}+V_{\text {tot }}(r)\right\} \varphi_{i}(\mathbf{r})=\varepsilon_{i} \varphi_{i}(\mathbf{r})$

Note that the entropy part of $G_{s}$ in (3) does not contribute to $(7)$, since the non-interacting entropy $S_{s}$ does not depend explicitly on the wavefunctions (see Sect. 2.2). In (7), $\hat{T}$ is the kinetic energy operator and the local potential $V_{\text {tot }}$ is a sum of three terms:

$V_{\mathrm{tot}}(r)=V_{I}(r)+V_{H}[\rho(r)]+V_{x c}[\rho(r)]$,

whereof $V_{I}$ is the jellium potential already mentioned, $V_{H}[\rho]$ is the Hartree potential of the electrons, given in the square brackets in (4) above, and the last term is due to the exchange and correlation contributions:

$$
V_{x c}\left[\rho([r)]=\frac{\delta}{\delta \rho(r)} \mathscr{F}_{x c}[\rho]\right.
$$

A word has to be said about the choice of the exchange-correlation free energy $\Omega_{x c}$ which depends, in principle, explicitly on the temperature (not only through $\rho$ ):

$\Omega_{x c}[\rho, T]=\int \mathscr{F}_{x c}[\rho(r), T] \mathrm{d}^{3} r$.

Gupta and Rajagopal [5] have calculated $\Omega_{x c}$ for uniform electron plasmas and presented it as a function of the reduced temperature $t=T / T_{F} . \Omega_{x c}$ was shwon to approach zero with increasing $t$, but only for $t \geqslant 0.1$ does a noticeable temperature dependence set in. The Fermi temperature $T_{F}$ itself depends on the density of the system like $T_{F} \sim \rho^{2 / 3}$. Using these results within the local density approximation (LDA), one therefore has a different reduced temperature $t$ at each point where the density $\rho(r)$ is varying. For the typical bulk electron densities of alkali metals $\left(\rho_{I} \simeq 10^{22}-10^{23} \mathrm{~cm}^{-3}\right)$, with which we are concerned here, $T_{F}$ is [5] of the order of $10^{4}-10^{5} \mathrm{~K}$, so that the temperature dependence of $\Omega_{x c}$ is practically negligible in the interiour of the clusters at temperatures below $2000 \mathrm{~K}$. Only in the extreme surface, where $\rho$ has decreased by two to three orders of magnitude, will the temperature variation of $\Omega_{x c}$ come into effect. But the contribution at low density to the total electronic free energy is small, and it seems therefore perfectly well justified to replace $\mathscr{F}_{x c}[\rho(r), T]$ in (4) by the $T=0$ energy density functional $\mathscr{E}_{x c}[\rho]$.

In conclusion, the temperature effects in alkali metal clusters can be expected to come only from the occupation numbers $n_{i}$, which will be determined in the following, and from the corresponding changes in the densities (5) and the mean field (8). In our numerical calculations, we used for $\mathscr{E}_{x c}[\rho]$ the LDA functional of Gunnarsson and Lundqvist [7].

In an exact treatment of the Coulomb exchange, the total potential $V_{\text {tot }}(r)$ would fall off asymptotically like $1 / r$ at large distances. The spectrum $\varepsilon_{i}$ would therefore contain an infinite number of bound Rydberg states which could lead the sums over the single-particle states $i$ to diverge. Due to the use of the LDA functional, however, the asymptotic fall-off of $V_{\text {tot }}$ is faster than $1 / r$ and there is only a finite number of bound states [2], so that this 
divergence problem is regularized automatically in the local density approximation.

We have solved (7) iteratively for spherical clusters on a finite mesh in $r$-space. The explicit form of the occupation numbers $n_{i}$ in terms of the single-particle spectrum $\varepsilon_{i}$, which have to be included at each iteration, depends on the choice of the statistical ensemble and will be discussed in the following subsection. For partially filled spherical shells we made the usual [2] 'filling approximation' which amounts to an averaging of the occupied states over their polar angles $(\theta, \phi)$, such that the density $\rho$ and the total potential $V_{\text {tot }}$ stay spherical.

\subsection{Entropy and occupation numbers}

We shall now discuss the calculation of the entropy and the occupation numbers at finite temperature $T$. As shown above, we need only know these quantities for a system of non-interacting Fermions in a local potential $V_{\text {tot }}(r)$, with eigenenergies $\varepsilon_{i}$ according to (7), at each given temperature. We shall, therefore, in the following omit the subscript $s$ of the entropy $S_{s}$. We call $U_{s}=\sum \varepsilon_{i} n_{i}$ the total internal energy of this system, such that $F_{s}=U_{s}-T S$ is its free energy. The energies $F_{s}$ and $U_{s}$ should not be confused with those of the interacting system, i.e., $F[\rho]$ and $U[\rho]$ in (2) and (4), which have entirely different values.

a) Grand canonical ensemble: We start with the grand canonical ensemble which is fairly standard and easy to calculate. The entropy for this ensemble is given explicitly in terms of the occupation numbers as [8]

$$
\begin{aligned}
S\left[n_{i}\right] & =\sum_{i} s\left(n_{i}\right) \\
& =-\sum_{i}\left\{n_{i} \log n_{i}+\left(1-n_{i}\right) \log \left(1-n_{i}\right)\right\} .
\end{aligned}
$$

Minimizing the free energy - non-interacting or interacting does not matter, as long as (7) is used - with respect to the $n_{i}$, using a constraint on the particle number $N$

$$
\sum_{i} n_{i}=N
$$

with the help of a Lagrange multiplier $\mu$

$$
\frac{\delta}{\delta n_{i}}\left\{F-\mu \sum_{j} n_{j}\right\}=0
$$

i.e., minimizing the grand potential $\Omega=F-\mu N$, leads to the Fermi occupation numbers

$n_{i}=\left\{1+\exp \left[\left(\varepsilon_{i}-\mu\right) / T\right]\right\}^{-1}$.

In the KS calculations, the chemical potential $\mu$ must be determined at each iteration such as to fulfil (12) and (14). The entropy $S$, (11), need only be calculated at the end, after convergence of the KS iterations.

It should be remembered that $N$ in (12) is only an average particle number in the grand canonical ensemble.
The variance of the particle number $N$ is given by

$\sigma_{N}^{2}=\sum_{i} n_{i}\left(1-n_{i}\right)=-T \sum_{i} \frac{\partial n_{i}}{\partial \varepsilon_{i}}$.

Even for moderate temperatures $\sigma_{N}$ is easily of order unity for the clusters considered in this paper. Therefore we have to investigate the canonical ensemble where $N$ is fixed exactly from the beginning.

b) Canonical ensemble: In order to calculate entropy and occupation numbers for a canonical ensemble, we cannot avoid evaluating the partition function $Z_{N}(\beta)$ which is given by [8]

$Z_{N}(\beta)=\sum_{\alpha} \mathrm{e}^{-\beta E_{\alpha}(N)} ;$

$\beta=1 / T$ is the inverse temperature. The sum runs over all partitions $\alpha$, i.e. all possibilities to distribute $N$ particles over the single-particle levels $\varepsilon_{i}$, with energies $E_{\alpha}(N)$ :

$E_{\alpha}(N)=\sum_{i} p_{i}^{\alpha} \varepsilon_{i}, \quad p_{i}^{\alpha}=0$ or $1, \quad \sum_{i} p_{i}^{\alpha}=N$.

From $Z_{N}(\beta)$ we get $U_{s}, F_{s}$ and $S$ by the canonical relations

$$
\begin{aligned}
& F_{s}=-\log Z_{N} / \beta, \\
& U_{s}=-\frac{1}{Z_{N}} \frac{\partial}{\partial \beta} Z_{N}, \\
& S=\beta\left(U_{s}-F_{s}\right) .
\end{aligned}
$$

To define the occupation numbers $n_{i}$, we start from the basic probability $P_{\alpha}$ for the system to have the energy $E_{\alpha}$ at the temperature $1 / \beta$ :

$P_{\alpha}=\mathrm{e}^{-\beta E_{\alpha}(N)} / Z_{N}(\beta) ; \quad \sum_{\alpha} P_{\alpha}=1$.

In terms of the $P_{\alpha}$, we can write the internal energy $U_{s}$ and the entropy $S$ as

$U_{s}=\left\langle E_{\alpha}\right\rangle=\sum_{\alpha} P_{\alpha} E_{\alpha}$,

$S=-\left\langle\log P_{\alpha}\right\rangle=-\sum_{\alpha} P_{\alpha} \log P_{\alpha}$.

The $n_{i}$ now are defined as the ensemble averages of the microscopic occupations $p_{i}^{\alpha}$ :

$n_{i}=\left\langle p_{i}^{\alpha}\right\rangle=\sum_{\alpha} P_{\alpha} p_{i}^{\alpha}$.

Combining (17), (19), (20) and exchanging sums, we see that

$\sum_{i} \varepsilon_{i} n_{i}=U_{s} ; \quad \sum_{i} n_{i}=N$.

In practice, the evaluation of $Z_{N}$ and the $n_{i}$ cannot be done by summing explicitly over all partitions $\alpha$ in (16) and (22), because there are far too many of them for 
$N \geq 10$. An economic way of calculating these quantities exactly, nevertheless, is described in the Appendix. Still, the numerical treatment of the canonical ensemble, in particular the evaluation of the $n_{i}$, is far more time consuming than for the grand canonical case. We have therefore developed a way to avoid the iterative determination of the canonical $n_{i}$ in the KS calculations, which shall be presented and tested in Sect. 3.2 below.

\section{Numerical tests and approximations}

\subsection{Comparison of the two ensembles}

As some of the most sensitive quantities to details of level structure and to the choice of the statistical ensemble, we investigate the first and second differences of the total interacting free energy $F$, (4), of a neutral cluster with respect to the number $N$ of atoms:

$$
\begin{aligned}
\Delta_{1} F(N) & =F(N-1)-F(N), \\
\Delta_{2} F(N) & =\Delta_{1} F(N)-\Delta_{1} F(N+1) \\
& =F(N+1)+F(N-1)-2 F(N) .
\end{aligned}
$$

We recall that the energy of the ions is included only in the jellium approximation at $T=0$; therefore the entropy part in these quantities is coming exclusively from the valence electrons.

The first difference $\Delta_{1} F(N)$ is related to the dissociation free energy $D_{N}$ of one neutral atom by

$D_{N}=\Delta_{1} F(N)+F(1)$,

where $F(1)$ is the free energy of a single atom. This latter quantity, $F(1)$, is certainly not correctly described in the jellium model; nevertheless, (25a) turns out to be a good estimate of the experimental dissociation energy (see Sect. 5.1). In any case, since $F(1)$ is a constant, we may consider the factor $\exp \left\{-\beta \Delta_{1} F(N)\right\}$ to be a measure for the stability of the cluster $N$ against evaporation of a monomer:

$\mathrm{Na}_{N} \rightarrow \mathrm{Na}_{N-1}+\mathrm{Na}_{1}$

(See the beginning of Sect. 5 for a further discussion on the relation of $\Delta_{1} F$ and $\Delta_{2} F$ to mass abundances in cluster beams.)

On the other hand, in a chemical equilibrium of three adjacent sodium clusters,

$\mathrm{Na}_{N-1}+\mathrm{Na}_{N+1} \leftrightharpoons 2 \mathrm{Na}_{N}$

the law of mass action expresses that $\Delta_{2} F$ and cluster concentration (or probability) $c_{N}$ are related approximately through

$$
\begin{aligned}
\Delta_{2} F(N) & \simeq-T \Delta_{2} \log c_{N} \\
& =T \log \left[\frac{c_{N}^{2}}{c_{N-1} c_{N+1}}\right],
\end{aligned}
$$

$F(N)$ being the total free energy of a cluster at unit probability for a given volume and temperature. The relation (25b) would be exact if the rotational, translational and vibrational degrees of freedom of the ions were included. Treating those degrees of freedom classically and assuming that the cluster is a sphere with a volume proportional to its mass, their contribution to the free energy is a smooth function of cluster size. More specifically [1], this part of the free energy is the sum of a volume and a logarithmic term in cluster size, and differentiating twice leaves an error of order $N^{-2}$; hence its omission from $\Delta_{2} F(N)$ is inconsequential.

In Figs. 1-3 we show $\Delta_{1} F$ and $\Delta_{2} F$ as functions of temperature for a series of neutral $\mathrm{Na}$ clusters. The solid lines are obtained for the canonical ensemble and the short-dashed lines are obtained for the grand canonical ensemble. For both ensembles, the KS equations (7) have been solved iteratively including the corresponding occupation numbers $n_{i}$, until convergence (i.e., selfconsistency of the field $V_{\text {tot }}$ ) was reached. We see that there are significant differences between the results obtained with the two ensembles, in particular in the physically interesting region around temperatures of a few hundred up to about 1000 degrees. The error introduced by the particle number non-conservation in the grand canonical treatment differs from case to case, depending sensitively on the shell structure in the single-particle spectrum $\varepsilon_{i}$ near the Fermi energy. In all cases, the error decreases for $T \geq 1000 \mathrm{~K}$ and becomes very small for $T \geq 2000 \mathrm{~K}$.

The linear behaviour of $\Delta_{1} F(T)$ and $\Delta_{2} F(T)$ near $T=0$, with slopes that are clearly different for the two ensembles, can easily be understood in terms of the degeneracies of the last occupied levels $\varepsilon_{i}$. Indeed, for $T \lesssim 200 \mathrm{~K}$, the smaller clusters can be considered to be perfectly cold: the occupation numbers of all completely filled levels $\varepsilon_{i}$ are identically one. If there are $v$ particles in the last level $\varepsilon_{0}$ and the degeneracy of this level (including spin factor 2) is $g$, then the exact microscopic
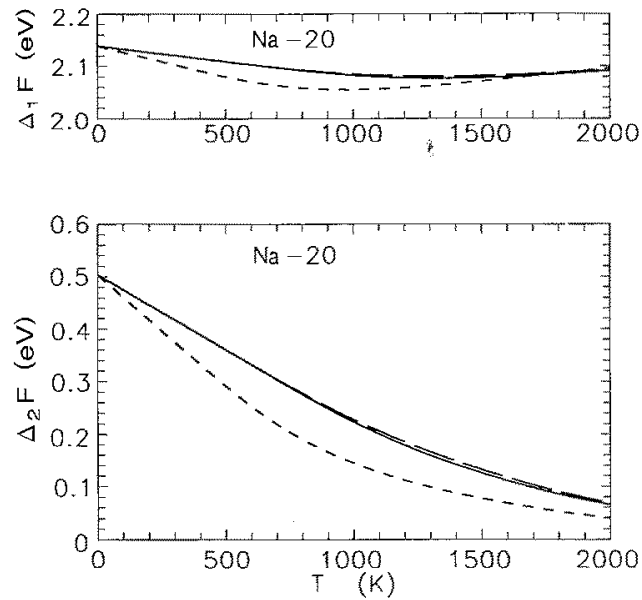

Fig. 1. First and second differences $\Delta_{1} F$ and $\Delta_{2} F(24)$, of total free energy of the sodium cluster with $N=20$ versus temperature. Solid lines: selfconsistent canonical results. Short-dashed lines: selfconsistent grand canonical results. Long-dashed lines: result of approximation (32), i.e. using one canonical iteration at the end of a grand canonical selfconsistent calculation 

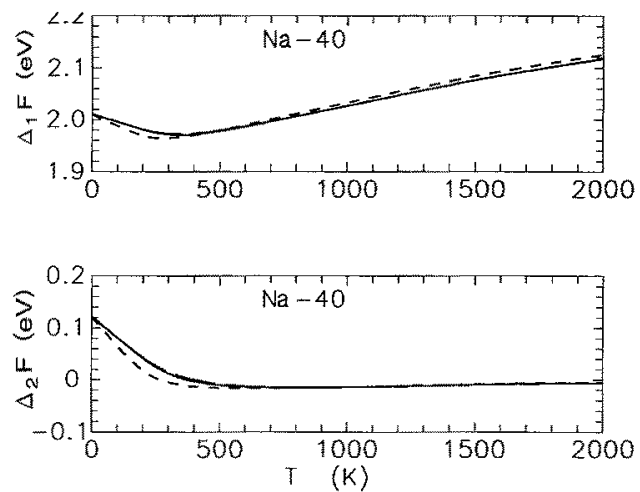

Fig. 2. As Fig. 1, for $N=40$
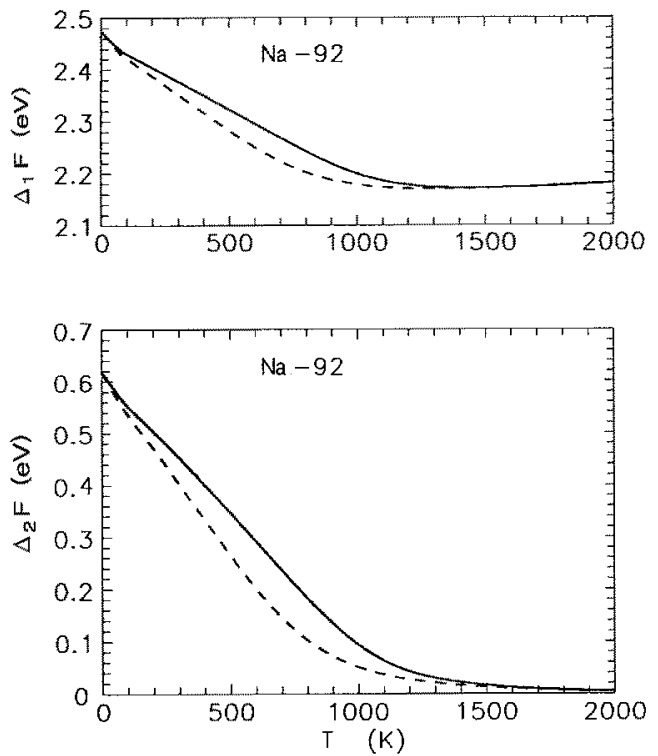

Fig. 3. As Fig. 1, for $N=92$

probability $P_{\alpha}$ and the occupation number $n_{0}$ of this state are given by

$P_{\alpha}=\left(\begin{array}{l}g \\ v\end{array}\right)^{-1}=\frac{v !(g-v) !}{g !} ; \quad n_{0}=\frac{v}{g}$.

The canonical entropy of the corresponding cluster in the limit $T=0$ is then, with (21),

$$
\begin{aligned}
S_{0}^{\mathrm{can}} & =\log \left(\begin{array}{l}
g \\
v
\end{array}\right) \\
& =\log g !-\log v !-\log (g-v) ! .
\end{aligned}
$$

(This value is exactly reproduced in our numerical calculation for the canonical ensemble from (16), (18) in the limit $T=0$.) Since the internal energy $U(T)$ has a zero slope at $T=0$, the slopes of $\Delta_{1} F$ and $\Delta_{2} F$ are thus identical to minus the first and second differences $\Delta_{1} S$ and $\Delta_{2} S$, respectively, of the entropy at $T=0$.

For the grand canonical ensemble, now, one obtains from (26) and (11) a different value of the entropy, namely

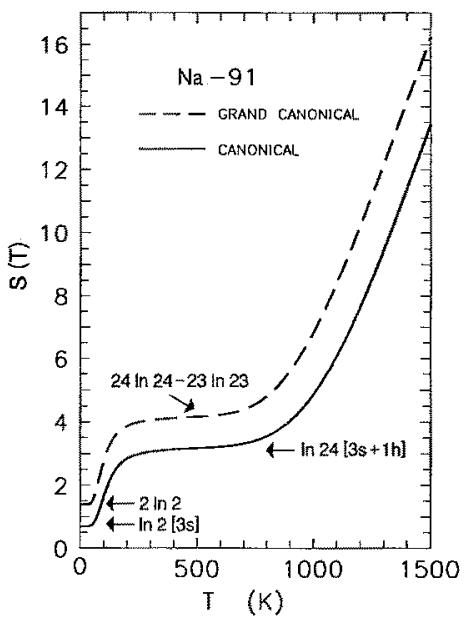

Fig. 4. Entropy of the $\mathrm{Na}_{91}$ cluster versus temperature. Solid line: canonical result, dashed line: grand canonical result. The finite values at $T=0$ come from one electron in the $3 \mathrm{~s}$ level (degeneracy $g=2$ ). The plateau values for $300 \leqq \mathrm{~T} \$ 600 \mathrm{~K}$ are the $T=0$ entropies of an electron in a 24 -fold degenerate level, corresponding to the combined $3 s+1 h$ levels of this cluster (see text)

$$
\begin{aligned}
S_{0}^{\text {g.c. }} & =s\left(n_{0}\right) \\
& =g \log g-v \log v-(g-v) \log (g-v)
\end{aligned}
$$

which, incidentally, corresponds to using Stirling's approximation to the factorials in the correct expression (27). In the case of $v=1$ particle in an $s$ level (angular momentum zero, spin degeneracy $g=2$ ), the error in (28) with respect to the canonical entropy (27) is a factor of two.

This is illustrated in Fig. 4 where we show the entropy of the $\mathrm{Na}_{91}$ cluster as a function of temperature, evaluated both for the canonical and the grand canonical ensemble. The highest occupied level in this cluster at $T=0$ is the $3 s$ level: we clearly recognize the two limiting values $S_{0}^{\text {can }}=\log 2$ and $S_{0}^{\text {s.c. }}=2 \log 2$ at the left of the figure. In the temperature region $300 \leqq T \lesssim 600 \mathrm{~K}$, the entropies in Fig. 4 exhibit plateaux at the values $S^{\text {can }}=\log 24$ and $S^{\text {g.e. }}=24 \log 24-23 \log 23$, respectively. This is due to the fact that the $3 \mathrm{~s}$ level is nearly degenerate with the $1 \mathrm{~h}$ level $(g=22)$ which lies lower in energy by only $0.033 \mathrm{eV} \simeq 400 \mathrm{~K}$. Therefore, at temperatures around $T \simeq 500 \mathrm{~K}$, these two levels appear as one level with a total degeneracy of $g=2+22=24$, whereas all the other levels of the spectrum are far enough away to have occupation numbers equal to 1 or 0 and thus do not contribute to the entropy. This rapidly changing increase of the entropy at small temperatures is another manifestation of electronic shell structure. Only above several thousand degrees $\mathrm{K}$, the shell effects are averaged out and $S$ takes on its expected linear temperature dependence (see Sect. 5.2).

We have thus found that the differences in the slopes of the curves in Figs. 1-3 at small $T$ reflect the wrong entropies obtained in the grand canonical treatment. Note that the error, near $T=0$, does not lie in the Fermi occupation number given by (14) which also leads to the 


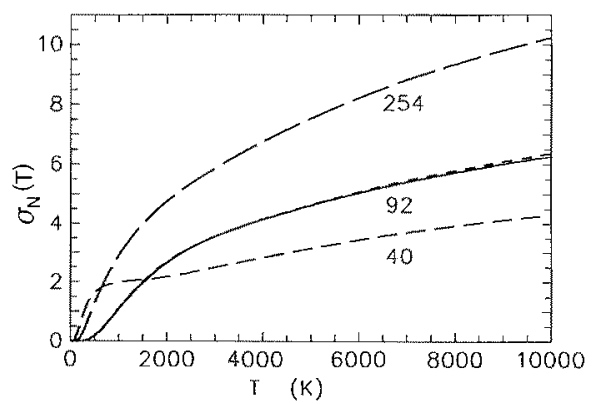

Fig. 5. Root of particle number variance $\sigma_{N}(15)$, versus temperature for the clusters with $N=40,92$, and 254 in the grand canonical treatment. Solid line: exact selfconsistent result. Dashed lines: using the approximation of Sect. 3.3 , in which the spectra $\varepsilon_{i}^{(0)}$ of the cold clusters $(T=0)$ are used

correct numerical value of $n_{0}(26)$ at small temperatures (although the exact $T=0$ limit does not exist for $\varepsilon_{0}=\mu$ ).

In Fig. 5 the variance $\sigma_{N},(15)$, of the particle number, obtained in the grand canonical ensemble for three cluster sizes is plotted versus temperature up to $T=10000 \mathrm{~K}$. For $N=92$, the solid line is the fully selfconsistent result. The dashed lines show $\sigma_{N}$ obtained in the approximation where the cold spectrum $\varepsilon_{i}^{(0)}$ of the selfconsistent solution at $T=0$ is used; an approximation which is seen here to work very well for $\sigma_{N}$. (See, however, the discussion and results in Sect. 3.3 below). From these results it is not surprising that errors enter into the differences $\Delta_{1} F(N)$ and $\Delta_{2} F(N)$, since they hinge precisely upon an exact knowledge of the particle number $N$.

For $T \geqslant 3000 \mathrm{~K}$, all three curves in Fig. 5 can be parametrized approximately by $\sigma_{N}^{2} / N \simeq c T$ with $c=0.045 \cdot 10^{-3} \mathrm{~K}^{-1}=7.1 \mathrm{Ry}^{-1}$. This linear $T$ dependence is easily derived from the rhs of (15), if shell effects are neglected and the average single-particle level density $\tilde{g}(\varepsilon)$ is kept constant to its value at the Fermi energy:

$$
\begin{aligned}
\sigma_{N}^{2} & \sim \int_{-\infty}^{\infty} \tilde{g}(\varepsilon) n_{\varepsilon}\left(1-n_{\varepsilon}\right) \mathrm{d} \varepsilon \\
& \simeq-T \tilde{g}\left(\varepsilon_{F}\right) \int_{-\infty}^{\infty} \frac{\partial n}{\partial \varepsilon} \mathrm{d} \varepsilon=T \tilde{g}\left(\varepsilon_{F}\right)=\frac{6}{\pi^{2}} a N T
\end{aligned}
$$

where $a$ is the level density parameter discussed in Sect. 5.2. The numerical value $a=11.6 \mathrm{Ry}^{-1}$ found there from fitting entropies to (46) is in quantitative agreement with $c=\frac{6}{\pi^{2}} a$, indeed.

Summarizing this subsection, we can state that the canonical treatment of the clusters under investigation here is mandatory in order to obtain quantitatively correct results, especially for the entropy. The spread in particle number in the grand canonical treatment is, in particular, too large to obtain exact values for the quantities $\Delta_{1} F$ and $\Delta_{2} F$ at the physically interesting temperatures below $T \simeq 1000 \mathrm{~K}$. Another quantity that is sensitive to a canonical treatment is the specific heat; it will be discussed in Sect. 5.3.

\subsection{Approximately selfconsistent treatment of the canonical ensemble}

The iterative inclusion of the canonical occupation numbers $n_{i}$ (see the Appendix) in the $\mathrm{KS}$ calculations is rather time consuming for large clusters and high temperatures. We have therefore developed an approximate scheme which we will show here to be completely sufficient for all practical purposes. It permits one to obtain the canonical results with only negligibly more computing time than that required for the grand canonical calculation.

Our idea is to assume that the main error in the grand canonical calculation does not lie in the determination of the occupation numbers $n_{i},(14)$, but in (11) for the entropy. In the limit $T=0$, we have just shown in Sect. 3.1 above that this is, indeed, exactly the case. Now, in the iterative solution of the KS equations (7), only the occupation numbers $n_{i}$ are needed for the density (5) and the entropy never enters. Thus, the temperature dependence of the selfconsistent mean field $V_{\text {tot }}$ in a grand canonical KS calculation is as good as the occupation numbers $n_{i}$ are. We therefore propose to perform the KS iterations with the grand canonical occupation numbers (14) until convergence is reached, and then to correct the total free energy perturbatively by introducing the canonical ensemble in a last step.

To do so, let us denote the exact canonical occupation numbers (2) by $n_{i}$ as before; the grand canonical ones, (14), (which become slightly wrong at $T>0$ ) by $\tilde{n}_{i}$, and their difference by $\delta n_{i}$ :

$\delta n_{i}=n_{i}-\tilde{n}_{i}$.

It is now straightforward, using the underlying variational principle which leads to the KS equations (7), to show that to first order in the differences $\delta n_{i}$, the error introduced in the total, grand canonical interacting free energy $F^{\text {s.c. }}=F\left[\rho, \tilde{n}_{i}\right](4)$ is equal to

$\delta_{1} F_{s}=F_{s}^{\mathrm{can}}-F_{s}^{\text {g.c. }}\left[\tilde{n}_{i}\right]$

in terms of the non-interacting free energies, evaluated for the two ensembles indicated by the superscripts, in terms of the level spectrum $\varepsilon_{i}$ of the grand canonical ensemble. Adding the correction $\delta_{1} F(30)$ to $F^{\text {g.c. }}$ will therefore give the selfconsistent canonical free energy $F^{\mathrm{can}}=F\left[\rho, n_{i}\right]$ up to errors of second and higher order in $\delta n_{i}$ :

$F^{\mathrm{can}}=F^{\mathrm{g} \cdot \mathrm{c} .}+\delta_{1} F_{s}+\mathscr{O}\left[\left(\delta n_{i}\right)^{2}\right]$,

so that we can use the approximation

$$
\begin{aligned}
F^{\mathrm{can}} & \simeq F^{\text {g.c. }}\left[\rho, \tilde{n}_{i}\right]+\delta_{1} F_{s} \\
& =U^{\text {g.c. }}\left[\rho, \tilde{n}_{i}\right]-\sum_{i} \varepsilon_{i} \tilde{n}_{i}+F_{s}^{\mathrm{can}} .
\end{aligned}
$$

Note that the entropy $S\left[\tilde{n}_{i}\right](11)$, which is the major cause for the error in the grand canonical ensemble, cancels on the rhs of (32) and is replaced by the canonical one (in $\left.F_{s}^{c a n}\right)$. Note also, that in the approximation (32) the canonical occupation numbers $n_{i}$ are not needed, since $F_{s}^{\mathrm{can}}$ is evaluated according to (18) directly from the ca- 
nonical partition function. Therefore, the partition function (16) has to be calculated only once at the end of the KS iterations, which takes only little extra computing time (see the Appendix) and thus makes our procedure a very efficient one.

This perturbative correction of the total energy of an interacting system, due to small differences in occupation numbers, has been applied many times in different fields of physics. It is the starting point of Landau's Fermi liquid theory [9] in terms of quasi-particles; in nuclear physics, it gives the basis of Strutinsky's shell-correction method [10] in the framework of Hartree-Fock (HF) theory (see also [11]), and in solid state theory a 'force theorem' has been derived along similar lines [12]. Finally, the Koopmans theorem which we shall discuss in Sect. 4 is also closely related to this line of arguments.

In Figs. 1-3, we have included the results obtained in the approximation (32) by the long-dashed lines. They are in all cases seen to reproduce the exact canonical results with a very small and practically negligible error. This result can be understood by looking at the singleparticle levels $\varepsilon_{i}$, which are almost identical for the two ensembles. In fact, also the mean field $V_{\text {tot }}$ is practically the same in both cases; this was the starting assumption for the derivation of the approximation (32).

Thus we have shown that the choice of ensemble is not essential for obtaining the selfconsistent mean fields and densities, and that for all practical purposes a perturbative treatment at the end of a grand canonical KohnSham iterative scheme is sufficient to describe the canonical ensemble.

\subsection{Importance of the selfconsistent temperature dependence of the mean field}

The question may be raised to which extent the temperature dependence of the self-consistent mean field $V_{\text {tot }}$, (8), is important in our calculations. In Fig. 6 we show the density $\rho(r)$ and the potential $V_{\text {tot }}(r)$ of the same cluster, obtained at the three temperatures $T=0,1000$ and $2000 \mathrm{~K}$ for the canonical ensemble. (The corresponding curves for the grand canonical ensemble would hardly be distinguishable on the scale of the figure.) We should like to mention that in HF calculations for hot nuclei, the dependence of the mean fields on temperature has been found earlier $[13,14]$ to be very weak.

In consequence, one may use arguments as those presented in Sect. 3.2 above to treat the finite temperature effects perturbatively at the end of a $\mathrm{KS}$ calculation at $T=0$. In fact, since the temperature dependence of the mean field only enters through the occupation numbers $n_{i}$, one may include the $T>0$ effects up to first order in $\delta n_{i}^{T}=n_{i}(T)-n_{i}(0)$ by adding the correction

$\delta_{T} F_{s}=F_{s}\left(T, \varepsilon_{i}^{(0)}\right)-\sum_{i=1}^{N} \varepsilon_{i}^{(0)}$

to the total ground state energy for $T=0$, leading to the approximation

$F\left[\rho, n_{i}\right] \simeq E[\rho, T=0]+\delta_{T} F_{s} ;$
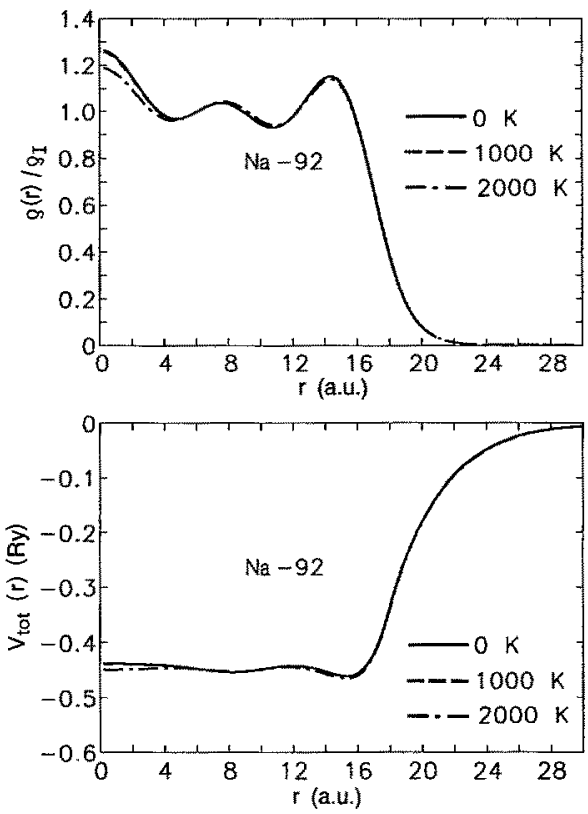

Fig. 6. Electron density $\rho(r)$, in units of jellium density $\rho_{I}$ (upper part), and total mean field $V_{\mathrm{tot}}(r)$ (lower part) of the $\mathrm{Na}_{92}$ cluster at three different temperatures, at $T>0$ evaluated for the canonical ensemble
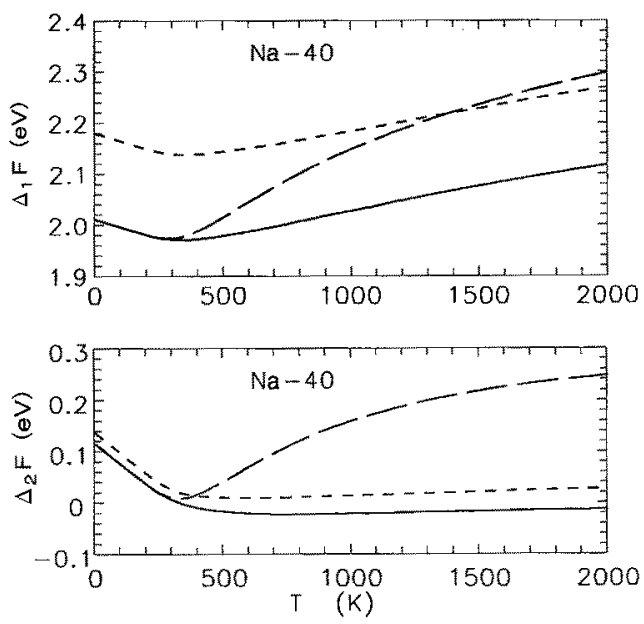

Fig. 7. Same as Fig. 2 for the canonical ensemble, but long-dashed lines: using the approximation (34) in terms of the cold specrum $\varepsilon_{i}^{(0)}$, and short-dashed lines: using the Koopmans approximation (43) discussed in Sect. 4. The solid lines are the exact selfconsistent results and identical to those in Fig. 2

hereby, the 'cold spectrum' $\varepsilon_{i}^{(0)}$ is used in (33). Equations (33), (34) may be used for either of the two ensembles discussed above. (In the nuclear HF calculations, done for grand canonical ensembles, this led to a fairly reasonable approximation of entropies and excitation energies [11]).

In Figs. 7, 8 we test the approximation (34) by comparing the results for $\Delta_{1} F$ and $\Delta_{2} F$, obtained from the $T=0$ spectrum $\varepsilon_{i}^{(0)}$ (long-dashed curves), to those of the fully selfconsistent canonical calculation (solid curves, identical to those in Figs. 2,3). For well-pronounced spherical magic shell situations, such as $N=92$, this ap- 

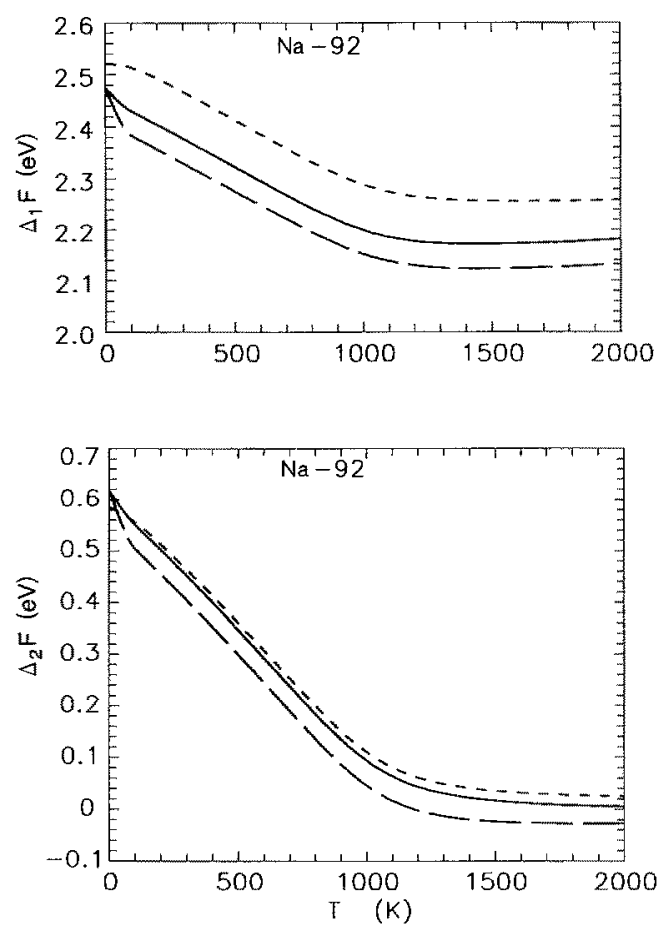

Fig. 8. As Fig. 7, for $N=92$

proximation is seen to work very well. However, in the case $N=40$, where there is only a weak gap in the spectrum $\varepsilon_{i}$, the temperature dependence of $\Delta_{1} F$ and $\Delta_{2} F$ obtained by (34) is not perfect. It therefore seems important to include the finite temperature selfconsistently in order to retreive correctly the details of shell structure in these quantities. In Sect. 4 we shall investigate the Koopmans theorem for $T>0$ and find a somewhat better perturbative description of the temperature dependence of $\Delta_{1} F$ and $\Delta_{2} F$.

\section{Discussion of the Koopmans theorem at $T>0$}

In this paper we put a great deal of emphasis on the quantities $\Delta_{1} F(N)$ and $\Delta_{2} F(N)$ which are closely related to separation energies of a neutral atom. At zero temperature, experience with the well-known Koopmans theorem in atomic, solid state [15] and nuclear physics [16] tells us that electron or nucleon separation energies can be well approximated by the Kohn-Sham (or HartreeFock) energy of the highest occupied single-particle level [17]. For the electronic ionization potential of a cluster with $N$ atoms at $T=0$, Koopmans' theorem reads [18]

$\mathrm{IP}(N)=-\varepsilon_{\mathrm{bo}}+\frac{e^{2}}{2 R_{I}}$,

where $\varepsilon_{\text {ho }}$ is the KS energy of the highest occupied level and the second term in (35) comes from the total electrostatic energy including the jellium self energy. Now, we can always rewrite $\Delta_{1} F(24 a)$ as

$$
\begin{aligned}
\Delta_{1} F(N) & =F(N-1)-F(N) \\
& =\operatorname{IA}(N-1)+\operatorname{IP}(N),
\end{aligned}
$$

where we have extended the definition of the ionization potential to finite temperatures by

$\operatorname{IP}(N)=F(N,-1)-F(N, 0)$

in terms of the free energy $F(N, z)$ of a cluster with $N$ atoms and $z$ excess electrons. In (36), IA $(N)$ is the ion affinity of a neutral cluster with $N$ atoms. This quantity is not expected to exhibit significant shell effects upon variation of $N$ - at least in the present jellium model. In our approximation (see Sect. 2.1) it also is independent of temperature. In order to estimate $\operatorname{IA}(N)$, we can therefore start from a semiclassical liquid-drop-model type expansion $[19,20]$ of the average energy $\widetilde{E}$ (at $T=0$ ) of a cluster with $N$ atoms and $z$ excess electrons:

$$
\begin{aligned}
\tilde{E}(N, z)= & E_{\text {Coul }}^{(0)}(N, z) \\
& -z \Delta \phi_{0}+e_{b}(N+z)+\ldots
\end{aligned}
$$

Here $E_{\text {Coul }}^{(0)}$ is the classical Coulomb energy of the cluster, corresponding to a square density distribution of the electrons. $\Delta \phi_{0}$ is the outer part of the Coulomb barrier of an infinite plane metal surface (i.e., its electrostatic potential taken between an infinite distance outside the metal and the jellium edge; see, e.g., [21]), $e_{b}$ is the electronic bulk energy (i.e., the energy per electron in the bulk metal), and the dots in (38) indicate surface energy and higher order terms which go at most like $N^{2 / 3}$ or $(N+z)^{2 / 3}$. (See [19] for the details of this expansion.) From (38), we find the leading order terms for the ion affinity

$$
\begin{aligned}
\operatorname{IA}(N) & =\tilde{E}(N, 0)-\tilde{E}(N+1,-1) \\
& \simeq-\Delta \phi_{0}-\frac{e^{2}}{2 R_{I}},
\end{aligned}
$$

the last term on the rhs of (38) coming from the classical Coulomb energy. Using (35) and (38) in (36), the terms $\frac{e^{2}}{2 R_{I}}$ cancel to leading order and we obtain the 'Koopmans approximation' for $\Delta_{1} F(N)$ at $T=0$ :

$\Delta_{1} E(N) \simeq-\varepsilon_{\mathrm{ho}}-\Delta \phi_{0}$.

The second difference then becomes straightforwardly

$\Delta_{2} E(N) \simeq \varepsilon_{1 \mathrm{u}}-\varepsilon_{\mathrm{ho}}$,

where $\varepsilon_{1 u}$ is the lowest unoccupied level in the cluster $N$ (or, to avoid confusion in case of degenerate levels: the highest occupied level in the cluster $N+1$; if a degenerate level is not completely filled in the cluster $N, \varepsilon_{\mathrm{lu}}$ and $\varepsilon_{\mathrm{ho}}$ will be identical and $\Delta_{2} E$ becomes zero). Note that the electrostatic correction $\Delta \phi_{0}$ and herewith all explicit contributions of the ions cancel in $\Delta_{2} E$ to leading order. 
Table 1. Test of approximations to first and second differences $\Delta_{1} E$ and $A_{2} E$, respectively, of total energies at $T=0$ for various cluster sizes $(N)$. a: Exact values, (1), evaluated from the selfconsistent interacting ground-state energy. b: Koopmans approximation, $(40,41)$. c: Using (43) in the limit $T=0$ with different spectra $\varepsilon_{i}$ for the different values of $N$

\begin{tabular}{|c|c|c|c|c|c|c|}
\hline \multicolumn{4}{|c|}{ Cluster $\Delta_{1} E(\mathrm{eV})$} & \multicolumn{3}{|c|}{$\Delta_{2} E(\mathrm{eV})$} \\
\hline & a & $b$ & $\mathrm{c}$ & a & $b$ & c \\
\hline 18 & 2.525 & 2.596 & 3.365 & 0.326 & 0.348 & -0.429 \\
\hline 19 & 2.199 & 2.241 & 3.794 & 0.060 & 0.000 & 0.055 \\
\hline 20 & 2.139 & 2.199 & 3.738 & 0.504 & 0.515 & 0.490 \\
\hline 21 & 1.635 & 1.784 & 3.248 & -0.096 & 0.000 & -0.003 \\
\hline 34 & 2.448 & 2.511 & 3.368 & 0.419 & 0.438 & -0.564 \\
\hline 39 & 2.017 & 2.165 & 3.964 & 0.006 & 0.000 & 0.017 \\
\hline 40 & 2.011 & 2.181 & 3.947 & 0.122 & 0.143 & 0.796 \\
\hline 92 & 2.474 & 2.521 & 4.150 & 0.617 & 0.586 & 0.085 \\
\hline 106 & 1.949 & 2.151 & 4.023 & -0.183 & -0.173 & 1.018 \\
\hline 254 & 2.354 & 2.458 & 3.866 & 0.272 & 0.271 & -0.539 \\
\hline 338 & 2.322 & 2.444 & 3.816 & 0.315 & 0.315 & -0.622 \\
\hline 339 & 2.008 & 2.131 & 4.439 & 0.001 & 0.000 & -0.004 \\
\hline 340 & 2.006 & 2.133 & 4.442 & 0.001 & 0.000 & -0.004 \\
\hline 450 & 2.113 & 2.239 & 2.878 & -0.002 & 0.000 & -0.005 \\
\hline 508 & 2.265 & 2.376 & 4.266 & 0.068 & 0.068 & 0.572 \\
\hline 832 & 2.243 & 2.388 & 3.619 & 0.046 & 0.047 & 0.912 \\
\hline
\end{tabular}

For later reference, we add here also the expression obtained from (38) for the average part (without shell effects) of the electronic ionization potential at $T=0$ :

$$
\begin{aligned}
\widetilde{\mathrm{IP}}(N) & =\tilde{E}(N,-1)-\tilde{E}(N, 0) \\
& \simeq \Delta \phi_{0}-e_{b}+\frac{e^{2}}{2 R_{I}} .
\end{aligned}
$$

(We have omitted here, as well as in (38), some minor contributions stemming from the missing surface energy terms in (38) which change the coefficient of $1 / R_{I}$ somewhat; in $\widetilde{\mathrm{IP}}$ the coefficient e.g. for Na clusters $\left(r_{s}=3.96\right.$ a.u.) becomes [19] $\sim 0.4$ instead of 1/2.) The first two terms on the rhs of (42) constitute a valid expression [21] for the work function of the bulk metal, $W=\Delta \phi_{0}-e_{b}$, which was shown in extended Thomas-Fermi variational calculations $[19,20]$ to be reached asymptotically by the ionization potentials of spherical clusters in the limit $N \rightarrow \infty$.

We show in Table 1 the results obtained in the 'Koopmans approximation' for $\Delta_{1} E$ and $\Delta_{2} E,(40,41)$, in columns $\mathrm{b}$. They are shown to reproduce the exact selfconsistent results (columns a) very well, within less than $0.15 \mathrm{eV}$ for $\Delta_{1} F$ and even much better for $\Delta_{2} F$, at all clusters sizes. The error in $\Delta_{1} F$ is furthermore approximately constant for the larger clusters and could be reduced by renormalizing the value of $\Delta \phi_{0}$. We have used the theoretical value for the infinite plane metal surface [19]: $\Delta \phi_{0}=0.63 \mathrm{eV}$ for $\mathrm{Na}$ with $r_{s}=3.96$ a.u. Note that the omission of this term in (40) would lead to very poor results for $\Delta_{1} F$.

By a straightforward extension of the derivation $[17,18]$ of the Koopmans theorem and the above considerations leading to (40) and (41), we find for $T>0$

$\Delta_{1} F(N) \simeq \Delta_{1} F_{s}(N)-\Delta \phi_{0}$,
This consists in replacing the interacting free energy $F$ by the non-interacting one, $F_{s}$, and adding the electrostatic correction $\Delta \phi_{0}$ (which may, in principle, depend on the temperature) to the first difference $\Delta_{1} F$. However, in taking the differences of $F_{s}(N)$, one must make sure to keep a fixed spectrum $\varepsilon_{i}$, i.e., not to use different spectra $\varepsilon_{i}$ for the neighbouring clusters $N$ and $N \pm 1$ ! Otherwise (43) would not reduce to $(40,41)$ in the limit $T \rightarrow 0$, since the sum of all fully occupied levels at $T=0$ no longer would cancel. (We shall test below what this would lead to.)

The extension of the 'Koopmans approximation' to $T>0$ therefore consists in using the non-interacting free energy $F_{s}(N)$, but taking the differences with respect to $N$ in (43) for the fixed spectrum $\varepsilon_{i}$ of the cluster with $N$ atoms. Since the practical interest of the Koopmans theorem is to avoid selfconsistent iterations beyond the evaluation of the ground-state solution, we have tested this approximation using the spectrum $\varepsilon_{i}^{(0)}$ of the cold cluster at $T=0$, i.e. treating the $T>0$ effects only perturbatively as in Sect. 3.3. Here we have also kept $\Delta \phi_{0}$ constant at its $T=0$ value. The results are shown by the short-dashed curves in Figs. 7,8. The quality of this approximation at $T=0$, already demonstrated in columns a and $\mathrm{b}$ of Table 1 , appears to persist more or less up to $T=2000 \mathrm{~K}$ in all cases. It is, in particular, better than that of the approximation studied in Sect. 3.3 (and shown by the longdashed curves), where the fully interacting ground-state energy was used.

The necessity of using the fixed spectrum $\varepsilon_{i}$ of the cluster $N$ shall be demonstrated by showing what happens otherwise. Using (43) with the different selfconsistent spectra $\varepsilon_{i}^{(0)}$ obtained for each value of $N$ leads to results which differ from those of the approximation (34) discussed in Sect. 3.3 only by a constant independent of $T$, namely the difference between the interacting groundstate energy $E[\rho, T=0]$ and the sum of the lowest occupied levels $\varepsilon_{i}^{(0)}$ (plus the constant $\Delta \phi_{0}$ in the case of $\left.\Delta_{1} F\right)$. It is thus sufficient to give the results at $T=0$; their temperature dependence is identical to that of the longdashed curves in Figs. 7,8. The results for $\Delta_{1} E$ and $\Delta_{2} E$ are included in Table 1 in the columns $c$. Their values are seen to be much worse than those obtained in the Koopmans approximation and not sufficient to reproduce even qualitatively the shell effects contained in these quantities.

This may be surprising, because at first sight one might expect to improve the approximation by including a certain amount of selfconsistency in letting the spectra $\varepsilon_{i}$ adjust themselves to the particle size. The situation is, however, exactly the reverse. Using the non-interacting energy expression with $N$ dependent spectra would be an inconsistent ad hoc prescription, whereas Koopmans' theorem, which is derived [17] consistently from a change of occupation numbers with fixed wave functions and $\varepsilon_{i}$, has a solid variational basis.

We emphasize this point in view of practical applications of the Koopmans approximation. Indeed, our results seem to encourage its use in connection with parametrized phenomenological potentials, such as the Nilsson model potential of [1] or a Woods-Saxon potential fitted to selfconsistent Kohn-Sham results [22], whose parameters depend explicitly on the cluster size $N$. But

$\Delta_{2} F(N) \simeq \Delta_{2} F_{s}(N)$. 
the correct use of the expressions (43) with a fixed spectrum should then be borne in mind.

\section{Thermal properties and stability of hot metal clusters}

A good estimate of the electronic free energies is a key to understanding cluster concentrations in equilibrium. It is also equally important for the understanding of the process of evaporation of neutral atoms from clusters at finite temperatures. Evaporation is usually described by statistical theories where level density considerations play a major role. To date, only the phonon degrees of freedom have been included [23, 24], parametrizing the electronic influence by a single number $D$. If it can be assumed that the transition state of the evaporation process is identical with the fully dissociated final state, $D$ is equal to the free separation energy $D_{N}$ defined in (25a). In ordinary chemical reactions, $D_{N}$ can be evaluated at $T=0$ because the electronic subsystem remains in its adiabatic non-degenerate ground state and does not contribute to the total entropy. In general, however, the free separation energies are temperature dependent. For the case of sodium clusters, we have demonstrated this dependence in Sect. 3, linking it (for temperatures $0<T \lesssim 500 \mathrm{~K}$ ) directly to the high degeneracy of the electronic singleparticle levels next to the magic-shell gaps.

Even though not completely understood, the electronic modifications to the evaporative decay constant seem to be of considerable importance. It is likely that the observed shell structure in abundance spectra from an adiabatic expansion source results from evaporation between the time of formation and the time of detection (compare, e.g., the shell structure in the abundance spectra of [1] and [24]). Recent experimental results [25] in the large cluster region $N \simeq 200-600$, obtained by an expansion source, corroborate this hypothesis, displaying the characteristic saw-tooth behaviour of the separation energies in contrast to the much more symmetric shapes expected from quasi-equilibrium. In the observed spectra, the magnitude of the shell structure decreases and the widths of the shell closings increase with cluster size. Both these features are expected to result if the electronic free energy is included in the evaluation of decay constants [26]. The similarity of our curves for $\Delta_{1} F(N)$, presented below in Sect. 5.1, to the abundance spectra of [25] is, indeed, striking (see also [6]).

In lack of dynamical evaporation calculations, we content ourselves here by presenting systematic results for neutral clusters in their equilibrium states over a large range of cluster sizes at various temperatures. All calculations were done for $\mathrm{Na}$ clusters using the WignerSeitz radius $r_{s}=3.96$ a.u. for the jellium background. Unless otherwise mentioned, the free energy $F(N)$ was calculated for canonical ensembles in the approximation discussed in Sect. 3.2.

\subsection{Temperature dependence of $F(N), \Delta_{1} F(N)$ and $\Delta_{2} F(N)$}

We shall first briefly discuss the temperature dependence of the total free energy $F(N)$ of neutral Na clusters. Since the main effect of temperature is to reduce the shell structure, rather than to affect the average properties, we extract the fluctuating part of the total interacting free energy $F(N)$. Like in nuclear physics [10], we define the shell-correction energy $\delta F(N)$ by

$\delta F(N)=F(N)-\tilde{F}(N)$.

$\widetilde{F}(N)$ is the average value of $F(N)$ which, by definition, does not contain any shell effects. It may be obtained either by a numerical energy averaging [10] or by semiclassical methods [11]. Since we only need an approximate determination of $\tilde{F}$ for the present discussion, we simply use here its liquid drop model (LDM) expansion at $T=0$ already discussed in Sect. 4 (cf. (38) for the neutral case $z=0$ ):

$\tilde{E}(N)=e_{b} N+a_{s} N^{2 / 3}+a_{c} N^{1 / 3}+a_{0}$.

In principle, one can obtain the asymptotic values of the LDM parameters $a_{i}$ from semi-infinite calculations $[19,20]$, but these are strictly valid only for very large clusters and more terms would be needed in (45) to correctly describe small clusters, too. As a compromise for a fit to clusters with $8 \lesssim N \lessgtr 1000$, we take the bulk energy $e_{b}=-0.1659$ Ry and the asymptotic surface energy [19] $a_{s}=0.0400 \mathrm{Ry}$ as fixed values and adjust the parameters $a_{c}$ and $a_{0}$ by a simple eye fit to the calculated KS results of $F(N)$, yielding $a_{c}=0.0787 \mathrm{Ry}$ and $a_{0}=-0.0735 \mathrm{Ry}$.

In Fig. 9 we show the shell-correction $\delta F(N)$ versus $N^{1 / 3}$ at the three temperatures $T=0,400$ and $600 \mathrm{~K}$. Note the sharp minima at the magic numbers $N=8,20, \ldots$ indicating an increased stability of the corresponding spherical clusters. A figure very similar to ours at $T=0$ was
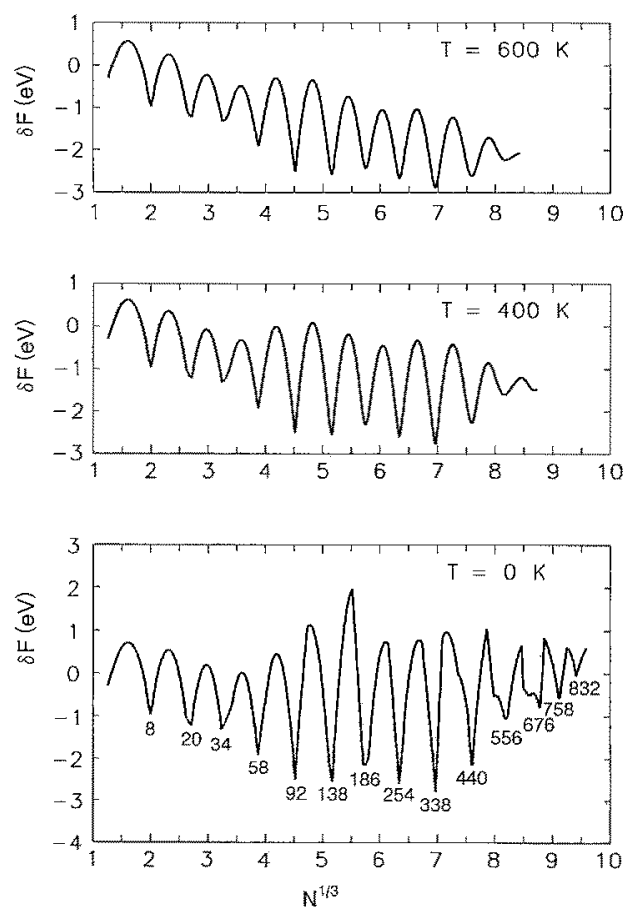

Fig. 9. Shell-correction $\delta F$ to the free energy, (44), versus particle number at three temperatures. The mag1c numbers corresponding to closed main shells are indicated for $T=0$ at the bottom 

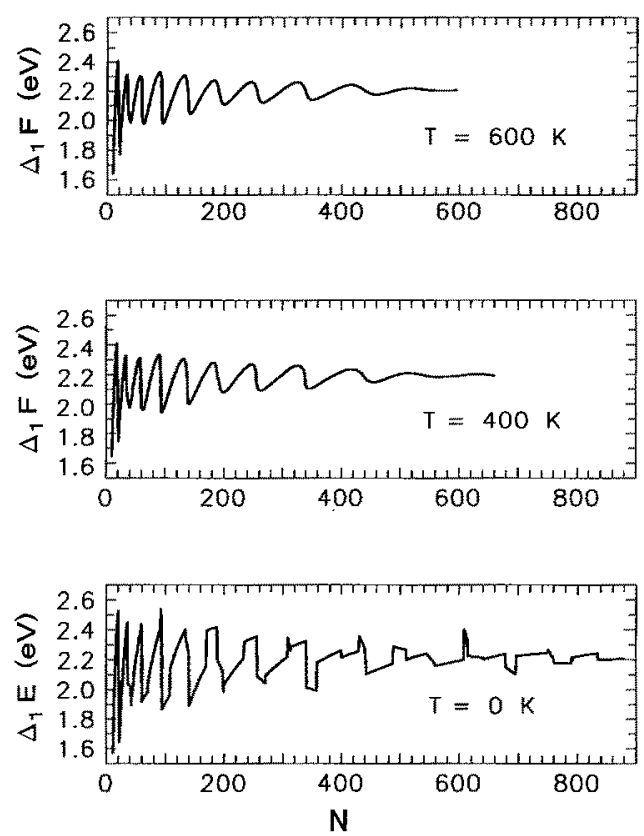

Fig. 10. First differences $\Delta_{1} F$ of free energies versus cluster size $N$ at three temperatures, evaluated for canonical ensembles in the approximation (32) in Sect. 3.2 (see text for details)

obtained in [22] with a phenomenological Woods-Saxon potential fitted to earlier KS potentials [2]. In the regions between the magic numbers, where $\delta F(N)$ has maxima, the clusters will be deformed like nuclei; therefore our absolute values of the shell-correction are exaggerated here due to the imposed spherical symmetry. The fact that $\delta F(N)$ at $T>0$ has a slight drift towards negative values for larger $N$ indicates that the correct LDM parameters should be temperature dependent. But the important thing to notice here is the decrease of the amplitude of $\delta F$ with increasing temperature for the larger clusters: for $N \sim 400-600$, even at the moderate temperature of $600 \mathrm{~K}$, it is reduced by a factor of at least 2 compared to its value at $T=0$. Also, the minima of $\delta F$ become less sharp with increasing cluster size and temperature. This effect will be emphasized when investigating the first and second differences of $F(N)$ in the following.

In Figs. 10 and 11 we present the quantities $\Delta_{1} F$ and $\Delta_{2} F$ as defined in (24a) and (24b), respectively, versus atom number $N \leqq 880$ at the same three temperatures. At $T=0$, we observe the familiar $[1,28]$ saw-tooth like behaviour of $\Delta_{1} F(N)$ and positive spikes of $\Delta_{2} F(N)$ at the magic numbers. The latter appear at the numbers $N=2$ and 8 (not shown), 18, 20,34, 40, 58, 92, (132), 138, 186, $196,254,(306,312), 338,(398,438), 440,508,(542,556)$, $612,(638), 676,758,(788)$, and 832 (minor shell closures in parentheses). Up to $N=196$, these magic numbers are in agreement with those calculated earlier [2] and with the older experimentally observed ones [1]. In recent experiments $[25,27]$ there is also evidence for magic shell closures of $\mathrm{Na}$ clusters around $N=260,344,440,558$, and, perhaps, a minor shell closure near $N \sim 394$.

The negative spikes seen in $\Delta_{2} F$ at $T=0$ are an artefact of the spherical symmetry imposed in our calculations.
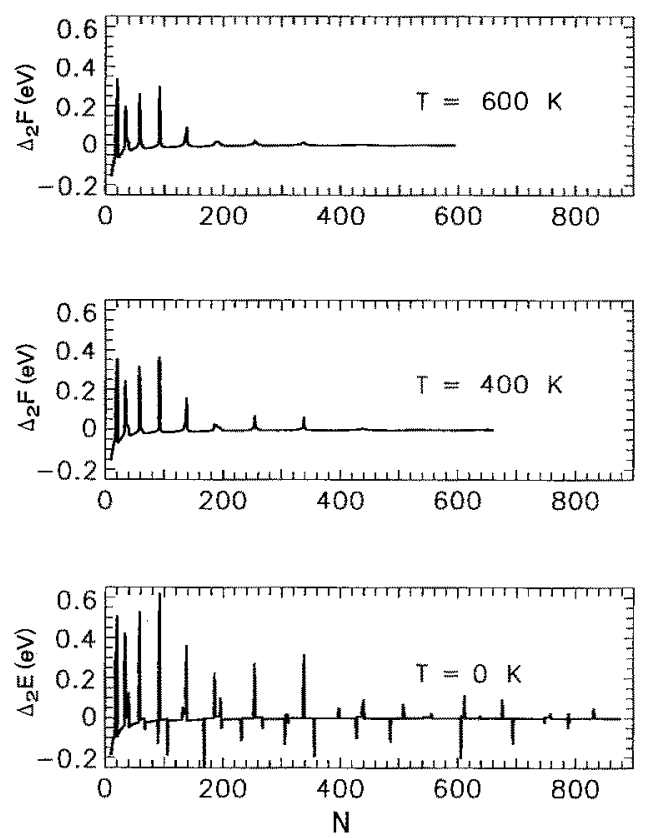

Fig. 11. As Fig. 10, but second differences $\Delta_{2} F(N)$

They appear when two levels lie very close to the Fermi energy. Due to their residual interaction, their positions change place during the filling of the lower state, such that when it is full, the originally higher one (assumed to stay empty) is shifted below the other. This is a typical selfconsistency effect which cannot occur with parametrized phenomenological potentials [1,22]. It happens in regions where we know that jellium clusters are deformed, like nuclei, once the spherical symmetry is relaxed (see also [28] for selfconsistent jellium model calculations for deformed clusters with $N \leqq 40$ ). In computing our figures, we chose to fill such pairs of levels sequentially (i.e., not allowing for two partially filled shells) in the order which leads to the lowest possible total energy for each particle number $N$. These negative spikes disappear as soon as a finite temperature is switched on. Due to the mixing of the spherical shells near the Fermi energy by the noninteger occupation numbers, such pairs of close levels are smeared over and appear like one degenerate larger shell; the energy then varies smoothly and monotonously with $N$ in the corresponding region.

The positive spikes in $\Delta_{2} F(N)$, on the other hand, are due to large gaps in the spectrum $\varepsilon_{i}$ at the Fermi energy, see (41). They survive the temperature averaging effects, as long as $T$ is considerably smaller than $\left(\varepsilon_{1 \mathrm{u}}-\varepsilon_{\mathrm{bo}}\right)$ of the magic cluster. However, as seen already in Sect. 3, their amplitudes decrease with increasing temperature. This effect, which goes along with a smear-out of the saw-tooth structure of $\Delta_{1} F(N)$, is seen in Figs. 10 and 11 to become more important with increasing cluster size. This is so because the spectra $\varepsilon_{i}$ become more compressed with increasing $N$.

The qualitative picture of our dissociation energies $\Delta_{1} F(N)$ at $T=400$ (or 600 ) $\mathrm{K}$ seen in Fig. 10 , with relatively sharp saw-teeth for $N \lesssim 50$ and their increasing 
smear-out and decreasing amplitude with increasing cluster size $N$, has an astonishing resemblance to the relative variations of the mass abundances of the recent experiments [25] around their average values. The global trend of the abundances cannot be compared because they depend largely on the dynamics of the ions, which is not included in our approach, and on the experimental conditions (pressure of the carrier gas, geometry of the nozzle, etc.). However, if one assumes that the relative variations are essentially due to the electronic shell effects in the free energy $F(N)$ - which is strongly supported already by the good agreement of the observed and calculated magic numbers in general -, this resemblance shows us that the finite entropy of the electronic system does play an important role and can, indeed, be observed!

From our analysis in Sect. 3.1 it becomes clear that it is essentially the entropy part $-T S$ of the electronic free energy that is responsible for the thermal smearing effects in $\Delta_{1} F(N)$ and $\Delta_{2} F(N)$. Detailed dynamical calculations including evaporation will, hopefully, make it possible to investigate more quantitatively in which way these two quantities determine the observed mass abundance spectra.

Our values of $\Delta_{1} F$ reach an average value of $\sim 2.2 \mathrm{eV}$ for large $N$. Indeed, according to (36), (38) in Sect. 4 , or also (45) above, their average value at $T=0$ should be $-e_{b}=2.26 \mathrm{eV}$ for $\mathrm{Na}$ clusters. This value is larger than the average experimental dissociation energy $D_{N} \simeq 1 \mathrm{eV}$ of a neutral atom. The difference is the binding energy of the neutral atom, $E(1)$, according to (25a). We cannot, of course, expect the jellium model to be correct for a single atom. Nevertheless, (25a) evaluated within the jellium model leads to an acceptable value of the average $D_{N}$. The total energy for $N=1$ obtained in our KohnSham calculation at $T=0$ is $E\left(\mathrm{Na}_{1}\right)=-1.06 \mathrm{eV}$; adding this constant to $-e_{b}$ gives, indeed, a very reasonable average dissociation energy of $D_{N}=1.2 \mathrm{eV}$. Thus, the main error in the total jellium binding energy, stemming from the neglect of the core electrons, cancels on the rhs of (25a) for the dissociation energy. Note from Fig. 10 that the average value of $\Delta_{1} F$ hardly depends on the temperature in the range considered here.

\subsection{Entropy and specific heat of the valence electrons}

In Fig. 4 in Sect. 3.1, we have already presented the temperature dependence of the total entropy of a finite cluster. At temperatures large enough to average out the shell effects, but still much smaller than the Fermi energy $\left(E_{F} \sim 2 \mathrm{eV} \sim 23000 \mathrm{~K}\right)$, one expects the entropy $S$ to be linear in $T$, i.e.

$S \sim 2 a N T$,

where $a$ is the level density parameter. For an infinite and homogeneous noninteracting Fermi gas, $a$ is given by $[8]$

$$
\begin{aligned}
a_{\infty} & =\frac{\pi^{2}}{6}\left(\frac{2}{3 \pi^{2}}\right)^{1 / 3}\left(\frac{2 m}{h^{2}}\right) r_{s}^{2} \\
& =0.670 r_{s}^{2}\left[\mathrm{Ry}^{-1}\right] .
\end{aligned}
$$

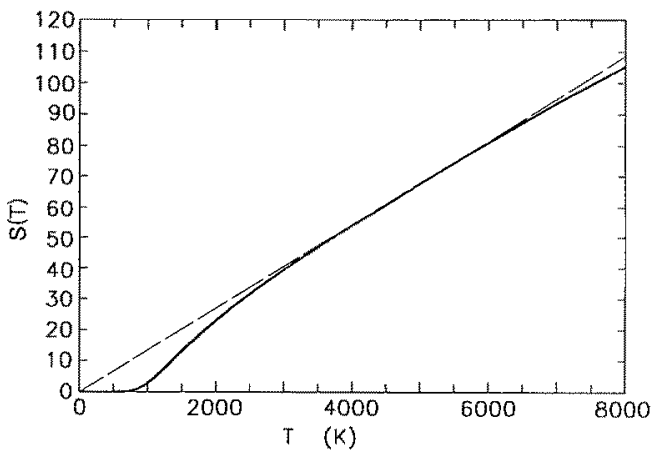

Fig. 12. Canonical entropy $S$ of the cluster $\mathrm{Na}_{92}$ versus temperature. The dashed line corresponds to the linear expression on the rhs of (46) with $a=11.6 \mathrm{Ry}^{-1}$. The deviation of this straight line below $T \simeq 3000 \mathrm{~K}$ is due to the shell effect (See text for deviation at large $T$ )

With $r_{s}=3.96$ a.u. according to $\mathrm{Na}$, this gives $a_{\infty}=10.5 \mathrm{Ry}^{-1}$.

In Fig. 12, we show the canonical entropy versus $T$, obtained for the $\mathrm{Na}_{92}$ cluster, up to very high temperatures. For $T \geqslant 3000 \mathrm{~K}$ it grows, indeed, linearly. (The slight deviation from a straight line above $T \sim 6000$ degrees is due to the fact that the number of states $\varepsilon_{i}$ included in the calculation was not sufficient; this is thus just a numerical truncation effect.) Fitting the slope in Fig. 12, we find $a=11.6 \mathrm{Ry}^{-1}$, in good agreement with the above Fermi gas value. The difference of about $10 \%$ is due to finite size corrections (e.g., a surface term in $a$ which would be proportional to $N^{-1 / 3}$ ). Note that the value of $a$ is identical for the grand canonical and the canonical ensemble; this has been confirmed in our results for various cluster sizes.

The temperature $T \simeq 3000 \mathrm{~K}$, at which the entropy in Fig. 12 reaches its homogeneous Fermi gas value (46), represents the critical temperature necessary to completely smear out the sheli effects. In heavy nuclei, this critical temperature was found [13] to be $\sim 3 \mathrm{MeV}$, which is about a third of a typical major shell spacing of the nucleonic single-particle levels. The situation is thus very similar here: $2500 \mathrm{~K} \simeq 0.2 \mathrm{eV}$ is about one third of the shell spacing at the Fermi level of the magic $\mathrm{Na}_{92}$ cluster (see Table 1). This is in good qualitative agreement with the result of a schematic analysis [29] in terms of a harmonic oscillator spectrum with level spacing $\hbar \omega$, for which the temperature dependence of the shell-correction energy of a closed-shell nucleus is found to be

$\delta F(T)=\delta F(0) \cdot \frac{t}{\operatorname{Sinh} t} ; \quad t=\frac{2 \pi^{2} T}{\hbar \omega}$.

Due to the factor $2 \pi^{2}$ in the dimensionless temperature variable $t$, a temperature $T \simeq \hbar \omega / 3$ is, indeed, sufficient here to suppress the $T=0$ value of the shell-correction by a factor of about 100 !

In Fig. 13. we plot the entropy $S / T N$ versus $N^{1 / 3}$ at the two temperatures $T=400$ and $600 \mathrm{~K}$. Dramatic shell effects are seen which essentially are due to the degeneracy of the spherical shells. As already observed in Sect. 3.1, up to temperatures of a few hundred degrees most smaller 

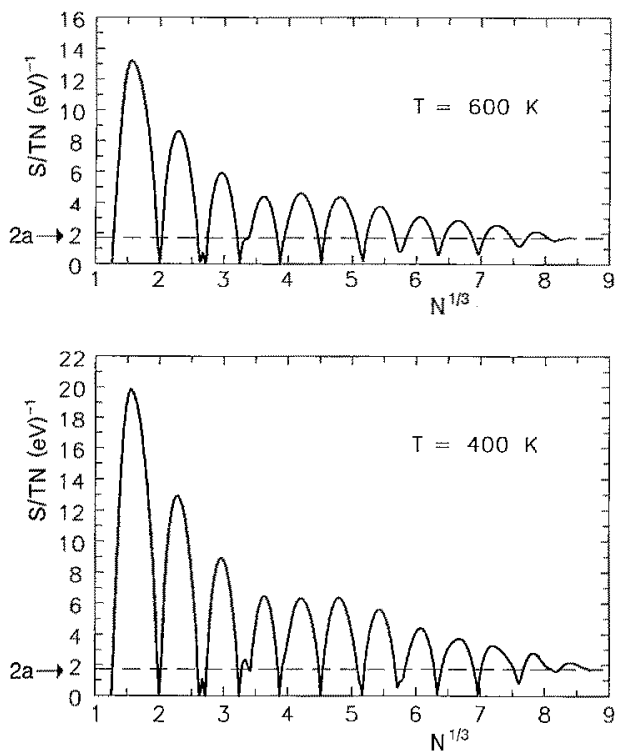

Fig. 13. Canonical entropy per particle, divided by $T$, at two temperatures, versus $N^{1 / 3}$. Note the decreasing amplitude of the shell oscillations with increasing temperature and particle number. The horizontal dashed lines indicate twice the level density parameter a according to (46)

clusters are essentially cold in the sense that their entropy still has its $T=0$ value $S_{0}$, which is given by (27) in terms of the occupancy of the highest (partially) filled shell. (The case $N=91$ shown in Fig. 4 is rather an exception due to the accidental near-degeneracy of two levels at the Fermi energy.) The oscillations in Fig. 13 therefore essentially represent the logarithms of the binomial coefficients in (27) for each region between two neighbouring filled shells (for which $S_{0}=0$ ). Only for larger temperatures and for larger sizes, a smearing of the entropy takes place; it is this effect which is responsible for the smearout of the differences $\Delta_{1} F$ and $\Delta_{2} F$ observed in Figs. 10 and 11. Note that the linear behaviour of $S$, (46), is reached also in the large- $N$ limit (at fixed $T$ ) with exactly the same value of the level density parameter $a$, as indicated by the horizontal dashed line in Fig. 13.

As a by-product of our calculations, we shall finally present some results for the electronic specific heat of metal clusters. The specific heat $c_{v}$ (here: heat capacity per electron) is defined as [8]

$c_{y}=\frac{1}{\mathrm{~N}} \frac{\partial U_{s}}{\partial T}=\frac{T}{N} \frac{\partial S}{\partial T}$

To keep the volume constant amounts here to work at fixed particle number $N$. For the canonical ensemble, we can rewrite this in terms of the partition function as

$c_{v}=\frac{1}{N} \beta^{2}\left\{\frac{1}{Z_{N}} \frac{\partial^{2}}{\partial \beta^{2}} Z_{N}-U_{s}^{2}\right\}$.

For the grand canonical ensemble, it is easiest to use the second expression in (48), taking the derivative $\partial S / \partial T$ at a constant chemical potential $\mu$. Note that in our present jellium model calculations, $c_{v}$ is a purely electronic
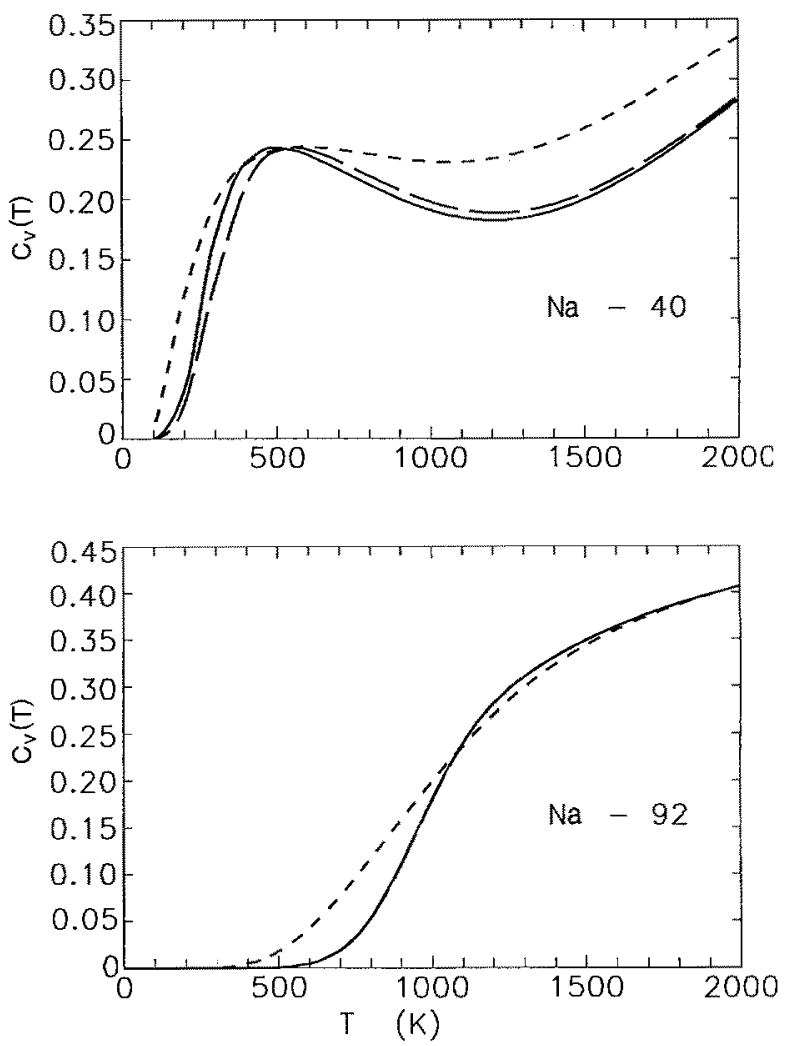

Fig. 14. Electronic part of the specific heat of the clusters $\mathrm{Na}_{40}$ and $\mathrm{Na}_{32}$ versus temperature. Solid lines: canonical results, short-dashed lines: grand canonical results, long-dashed lines (for $\mathrm{Na}_{92}$ hardly distinguishable from solid line!): canonical results in the approximation (32) discussed in Sect. 3.2

quantity since we have not included any ionic contribution to the entropy.

In Fig. 14 we show the electronic specific heat of the sodium clusters with $N=40$ and 92 , plotted versus temperature. As in Figs. 1-3, the solid lines show the canonical and the short-dashed lines the grand canonical results. They are seen to be quite different in $\mathrm{Na}_{40}$ even for $T \sim 2000 \mathrm{~K}$. The long-dashed curves are obtained in the perturbative treatment discussed in Sect. 3.2, which amounts to evaluating $c_{v}$ canonically in terms of the spectrum $\varepsilon_{i}$ obtained in the grand canonical KS calculation. This approximation is again seen to be satisfactory at all temperatures. Note the strong shell effects in the form of oscillations around the average (linear) increase with $T$, which are, of course, just a consequence of the shell structure in the entropy already discussed.

\section{Summary and conclusions}

We have presented microscopic density functional calculations of Kohn-Sham type for a canonical ensemble of metal clusters at finite temperatures, employing the jellium model for the description of the ionic background. The electronic shell effects, known from earlier calculations at $T=0$, are demonstrated to be smeared out by the finite temperature. 
We have, in particular, investigated the first and second differences, $\Delta_{1} F$ and $\Delta_{2} F$, of the free energy with respect to the number of atoms $N$. The quantity $\Delta_{1} F$ is likely to be related to the stability of clusters against evaporation of an atom and may thus be correlated with the observable mass abundance spectra. The shell fluctuations in these quantities are exclusively due to the valence electrons, the ionic background only giving smoothly varying contributions. A striking similarity of these shell fluctuations in our calculated curves $\Delta_{1} F(N)$ around $T \sim 400-600 \mathrm{~K}$ with the oscillations in recently measured [25] mass abundance spectra is found [6]. The smearing of the saw-tooth like structures and the reduction of their amplitude, which is clearly observable for the larger clusters with $N \geqslant 200$, are coming from the finite entropy of the valence electrons and its variation with temperature and cluster size.

The larger the clusters, the smaller are the shell spacings of the electron orbits and the larger is therefore the smearing effect of temperature. For temperatures $T \sim 400-600 \mathrm{~K}$, the smallest sizes with $N \lesssim 50$ are still cold as far as their valence electrons are concerned, such that the sharp saw-tooth structure in the dissociation energies or the peaks in their differences $\Delta_{2} F$ still persist. However, for large clusters with $N \geqslant 600$, very little shell structure is left at these temperatures. One might therefore question the practical feasability of observing the 'supershells', predicted [22] from $T=0$ considerations, using abundance measurements from adiabatic expansion sources of the type used in [25]. Spectroscopic techniques (see, e.g. [27]) which are directly sensitive to the position of the electronic single-particle levels $\varepsilon_{i}$ might be more favourable to this purpose. As we have found, these positions do not depend visibly on temperature.

The critical temperature which is sufficient to completely average out the shell effects has found to be $\sim 3000 \mathrm{~K}$ in a $\mathrm{Na}$ cluster with $N \sim 100$. Like in the case of nuclei, this is only about one third of the major shell spacing in the single-particle levels responsible for the shell effects. On the other hand, the total mean field of this cluster (and thus the positions of the single-particle energies) remain practically unaffected even up to temperatures of $T \geqslant 2000 \mathrm{deg}$. This means that all the averaging effects of temperature are brought about through the occupancies of the single-particle states alone. This statement, of course, only holds as long as the deformation of the mean field is kept constant, as in the present spherical calculations. Otherwise, the attenuation of the shell effects, which are responsible for deformed groundstate shapes in regions between the magic numbers, can lead to considerable variations of the mean fields accompanied by shape transitions (cf., again, the case of nuclei $[11,13])$, which we expect here to take place at temperatures $1000 \mathrm{~K} \lesssim T \lesssim 3000 \mathrm{~K}$.

As a consequence of the temperature independence of the mean spherical field, we have found that the Koopmans theorem can be exploited to extract finite-temperature results from the single-spectra of the cold clusters, obtained selfconsistently at $T=0$, in a quite reasonable approximation. Similarly, we have seen that if a selfconsistent Kohn-Sham calculation is wanted at finite tem- perature, the numerically much simpler grand canonical treatment is sufficient to yield the selfconistent mean field, from which the canonical calculation of the free energy, entropy and other thermodynamical quantities can be obtained perturbatively in a single iteration. Either of these two approximations might prove useful in future large-scale applications of the present method.

Like the mean field, we found also the average bulk energy $e_{b}$ to be nearly independent of temperature. Let us just briefly mention here that, using a local-current approximation to the random phase approximation (RPA) recently developed [30], we have also investigated the temperature dependence of the static dipole polarisabilities $\alpha_{s}$ and the frequencies of dipole plasmons of alkali clusters. We found both quantities to remain constant within a few percent up to $T \geqslant 2000 \mathrm{~K}$. This is not so surprising in the light of the above results and knowing [1] that $\alpha_{s}$ is roughly proportional to the volume of the cluster and thus a tpyical bulk quantity. Its deviation from the classical bulk value in smaller clusters, as well as the red-shift of the surface plasmon, is due to finitesize effects and therefore rather a surface than a bulk effect. Nevertheless, also this surface effect has by and large a smooth $N$ dependence, depending very little on local shell structure in the electron spectrum $\varepsilon_{i}$ and thus on temperature averaging effects.

We finally recall that we have imposed spherical symmetry in our calculations. Consequently, the role of shell effects is, to some extent, exaggerated in the present results. Nevertheless, we believe that the temperature smearing effects described here at and near the magic main shell closures are realistic, since the corresponding clusters are believed to be truly spherical and to stay so also when deformational degrees of freedom are taken into account. A closer examination of this aspect is in progress.

We are grateful to $\mathrm{S}$. Bjørnholm for his continuing interest and support, for a careful reading of the manuscript and many valuable comments. We also thank J. Borggreen, B. Mottelson, H. Nishioka and $J$. Pedersen for stimulating discussions. Two of us (M.B. and O.G.) acknowledge the Hospitality of the Niels Bohr Institute during several visits. The assistance of $\mathrm{G}$. Fuchs at the Rechenzentrum of the Regensburg University in accelerating the canonical ensemble code was a great help for handling the 'fat' clusters.

\section{Appendix}

\section{A. Calculation of canonical partition function and occupation numbers}

We want to calculate the partition function $Z_{N}(\beta)$ for distributing $N$ particles over $M$ levels with energy $\varepsilon_{i}-$ which we count such that each of the $\varepsilon_{i}$ is singly degenerate - at the temperature $T=1 / \beta$. Let us display the dependence on the number $M$ of levels explicitly (although it should numerically not depend on $M$ !):

$Z_{N}(\beta)=Z(N, M ; \beta)=\sum_{\alpha=1}^{I_{N M}} \mathrm{e}^{-\beta E_{\alpha}(N)}$ 
with $E_{\alpha}(N)$ given by the sum of occupied levels $\varepsilon_{i}$ for each partition $\alpha$ :

$E_{\alpha}(N)=\sum_{i=1}^{M} p_{i}^{\alpha} \varepsilon_{i} \quad$ with $\quad p_{i}^{\alpha}=0$ or $1 ;$

$\sum_{i=1}^{M} p_{i}^{\alpha}=N$

The number $I_{N M}$ of partitions is equal to a binomial coefficient

$I_{N M}=\left(\begin{array}{l}M \\ N\end{array}\right)$

and is usually huge. As a typical example, let us take the spherical cluster with $N=92$ electrons: to have a good convergence of $S$ at temperatures up to $\sim 2000 \mathrm{~K}$, we use 42 spherical levels; counting separately their degeneracies gives $M=338$ states and one finds $I_{N M} \sim 10^{86}$.

It is thus impossible, even on a large computer, to sum over the partitions $\alpha$ explicitly, except in very small clusters $(N \lesssim 10)$. In order to calculate, nevertheless, the canonical partition function (A1) and the derived thermodynamical quantities exactly, we use the following recurrence relation (which simply expresses the fact that the last level added is either occupied or unoccupied)

$$
\begin{aligned}
Z(N, M ; \beta)= & Z(N, M-1 ; \beta) \\
+ & \exp \left(-\beta \varepsilon_{M}\right) Z(N-1, M-1 ; \beta) \\
& (N \geqq 1, M \geqq N)
\end{aligned}
$$

with the extra conditions

$Z(0, M ; \boldsymbol{\beta}) \equiv 1, \quad \forall M \geqq 0 ;$

$Z(N, N-1 ; \beta) \equiv 0, \quad \forall N \geqq 1$.

Starting with $N=1$ particle, (A4) gives the one-particle partition function

$Z(1, M ; \beta)=\sum_{m=1}^{M} \mathrm{e}^{-\beta \varepsilon_{m}}$.

By adding successively more particles, one sees easily by induction that (A4) leads, indeed, to the correct $N$-particle partition function $Z(N, M ; \beta)$ obeying (A1) and (A2). For that, one thus has to perform the algebraic manipulation in (A4) - two multiplications, one exponentiation and one addition - only a number of $\tilde{I}_{N M}$ times, which is given by

$\tilde{I}_{N M}=N(M-N)$.

In the above example with $N=92$ and $M=338$, this gives $\tilde{I}_{N M}=22632$ instead of the $I_{N M} \sim 10^{86}$ exponentiations, multiplications and additions necessary to get $Z(N, M ; \beta)$ from (A1).
To get the derivatives of $Z$ with respect to $\beta$, needed for $U, S$ and $c_{v}$ in (18) and (49), we use the correspondingly derived forms of the recurrence relation (A4).

The evaluation of the occupation numbers $n_{i}$ in (22) is done similarly using the above recurrence scheme, by recalculating $Z(N, M ; \beta)$ for each level $\varepsilon_{i}$ in the situation where this level is kept empty (i.e. $\varepsilon_{i}$ is removed from the spectrum), dividing by the total $Z$ and subtracting the result from 1 . This calculation has to be done as many times as there are different (degenerate) levels $\varepsilon_{i}$ (42 in the above example). It takes, of course, much more computing time than in the grand canonical ensembles, but is still manageable for $N$ up to a few hundred and $T$ up to $\sim 10000 \mathrm{~K}$.

To check the numerical convergence we have ensured that all thermodynamical quantities derived from $Z$ and the $n_{i}$ did not depend on the number $M$ of states included in the calculations. Furthermore, the fraction $N_{\text {cont }}$ of electrons occupying the continuum region $(E>0)$ was found to be smaller than 0.1 up to $T \simeq 6000 \mathrm{~K}$, and well below unity even for $T \simeq 10000 \mathrm{~K}$. We can therefore be assured that for the physically interesting temperatures experienced by metal clusters, the electronic continuum effects play a completely negligible role.

\section{References}

1. Knight, W.D., Heer, W.A. de, Clemenger, K.: Solid State Commun. 53, 445 (1985); Heer, W.A. de, Knight, W.D., Chou, M.Y., Cohen, M.L.: Solid State Phys. 40, 93 (1987)

2. Ekardt, W.: Phys. Rev. B29, 1558 (1984); Beck, D.E.: Phys. Rev. B30, 6935 (1984)

3. Kohn, W., Sham, L.J.: Phys. Rev. 140, 1133A (1965)

4. Brack, M., Genzken, O., Hansen, K.: Z. Phys. D - Atoms, Molecules and Clusters 19, 51 (1991)

5. Mermin, N.D.: Phys. Rev. 137, 1441 A (1965); Gupta, U., Rajagopal, A.K.: Phys. Rep. 87, 259 (1982); Evans, R.: Adv. Phys. 28, 143 (1979)

6. Bjørnholm, S., Borggreen, J., Echt,. O., Hansen, K., Pedersen, J., Rasmussen, H.D.: Z. Phys. D - Atoms, Molecules and Clusters 19, 47 (1991)

7. Gunnarsson, O., Lundqvist, B.I.: Phys. Rev. B13, 4274 (1976)

8. See any textbook on Statistical Mechanics. We liked particularly well the presentation of $P$. Morse: Thermal Physics, Chs. $16 \mathrm{ff}$. New York: Benjamin 1965

9. Landau, L.: Sov. Phys. JETP 3, 920 (1957)

10. Strutinsky, V.M.: Nucl. Phys. A122, 1 (1968)

11. Brack, M., Quentin, P.: Nucl. Phys. A361, 35 (1981)

12. Mackintosh, A.R., Andersen, O.K.: In: Electrons at the Fermi Surface, Springford, M. (ed.). Cambridge: Cambridge University Press 1980

13. Brack, M., Quentin, P.: Phys. Lett. 52B, 159 (1974); Phys. Scr. A10, 163 (1974)

14. Bonche, P., Levit, S., Vautherin, D.: Nucl. Phys. A436, 265 (1985)

15. Perdew, J.P.: In: Density Functional Methods in Physics, p. 309. Dreizler, R.M., Providência, J. da (eds.) New York: Plenum Press 1985

16. Bassichis, W.H., Strayer, M.R.: Ann. Phys. (N.Y.) 66, 457 (1971) 
17. Janak, J.F.: Phys. Rev. B18, 7165 (1978)

18. Perdew, J.P.: In: Condensed Matter Theories, Vol. 4, p. 149. Keller, J. (ed.). New York: Plenum Press 1989

19. Seidl, M., Spina, M.E., Brack, M.: Z. Phys. D - Atoms, Molecules and Clusters 19, 101 (1991); see also Seidl, M., Meiwes-Broer, K.H., Brack, M.: J. Chem. Phys. 1991 (in press)

20. Spina, M.E., Seidl, M., Brack, M.: In: Symposium on atomic and surface physics - SASP '90, Märk, T.D., Howorka, F. (eds.), Innsbruck University, Austria, 1990, p. 426; see also: Brack, M.: Phys. Rev. B39, 3533 (1989)

21. Mahan, G.D., Schaich, W.L.: Phys. Rev. B10, 2647 (1974)

22. Nishioka, H., Hansen, K., Mottelson, B.R.: Phys. Rev. B42, 9377 (1990)

23. Engelking, P.C.: J. Chem. Phys. 87, 936 (1987)

Note added in proof. Sodium clusters with $N$ up to $\sim 3000$ have now been produced experimentally in an expansion source [Pedersen, J., Bjørnholm, S., Hansen, K., Martin, T.P., Rasmussen, H.D.: Preprint NBI-91-22. Nature (submitted for publication)] and the 'supershell' structure predicted in [22] has been clearly put into evidence-against the pessimism expressed in our summary (Sect. 6). We have extended our Kohn-Sham calculations and find a good qualitative agreement with the observed structure. If we multiply the calculated $\Delta_{2} F$ with a factor $N^{1 / 2} \exp \left(c N^{1 / 3}\right)$, as it was done with the logarithmic differences $\Delta_{1} \ln I_{N}$ of the experimental mass yields by Pedersen et al., we find, indeed, that the second supershell starting at $N \geq 850$ becomes visible even at $T \sim 500-600$ degrees Kelvin with an increasing amplitude of the shell oscillations [Genzken, O., Brack, M. (to be published)].
24. Bréchignac, C., Cahuzac, Ph., Leygnier, J., Weiner, J.; J. Chem. Phys. 90, 1492 (1989)

25. Bjørnholm, S., Borggreen, J., Echt, O., Hansen, K., Pedersen, J., Rasmussen, H.D.: Phys. Rev. Lett. 65, 1627 (1990)

26. Hansen K., et al.: (in preparation)

27. Martin, T.P., Bergmann, T., Göhlich, H., Lange, T.: Z. Phys. D - Atoms, Molecules and Clusters 19, 25 (1991) and submitted to Chem. Phys. Lett

28. Ekardt, W., Penzar, Z.: Phys. Rev. B38, 4273 (1988)

29. Bohr, A., Mottelson, B.: Nuclear Structure II, p. $607 \mathrm{ff}$. New York: Benjamin 1975

30. Reinhard, P.G., Brack, M., Genzken, O.: Phys. Rev. A41, 5568 (1990) 